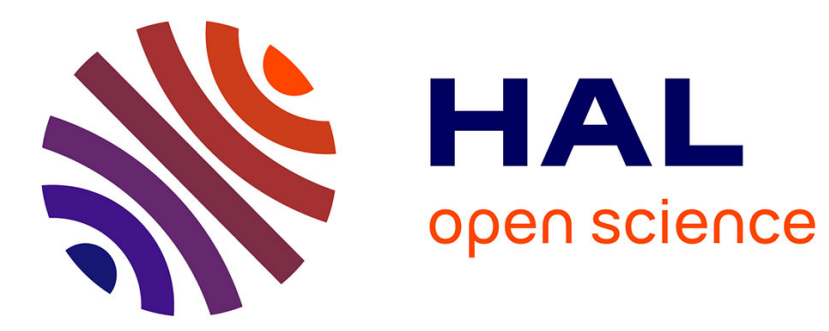

\title{
Biogeographic drivers of community assembly on oceanic islands: The importance of archipelago structure and history
}

Robin Aguilée, Félix Pellerin, Maxence Soubeyrand, Jeremy Choin,

Christophe Thébaud

\section{To cite this version:}

Robin Aguilée, Félix Pellerin, Maxence Soubeyrand, Jeremy Choin, Christophe Thébaud. Biogeographic drivers of community assembly on oceanic islands: The importance of archipelago structure and history. Journal of Biogeography, 2021, 48 (10), pp.2616 - 2628. 10.1111/jbi.14228 . hal03378117

\section{HAL Id: hal-03378117 \\ https://hal.science/hal-03378117}

Submitted on 14 Oct 2021

HAL is a multi-disciplinary open access archive for the deposit and dissemination of scientific research documents, whether they are published or not. The documents may come from teaching and research institutions in France or abroad, or from public or private research centers.
L'archive ouverte pluridisciplinaire $\mathbf{H A L}$, est destinée au dépôt et à la diffusion de documents scientifiques de niveau recherche, publiés ou non, émanant des établissements d'enseignement et de recherche français ou étrangers, des laboratoires publics ou privés. 


\title{
Biogeographic drivers of community assembly on oceanic islands: the importance of archipelago structure and history
}

\author{
Robin Aguilée ${ }^{\mathrm{a}, 1}$, Félix Pellerin ${ }^{\mathrm{b}}$, Maxence Soubeyrand ${ }^{\mathrm{c}}$, \\ Jeremy Choin ${ }^{\mathrm{d}, \mathrm{e}}$ and Christophe Thébaud ${ }^{\mathrm{a}}$ \\ Published in Journal of Biogeography, 2021, 48(10): 2616-2628 \\ doi: $10.1111 /$ jbi.14228
}

${ }^{a}$ Université Paul Sabatier Toulouse III, CNRS, IRD, UMR 5174 EDB Laboratoire Évolution \& Diversité Biologique, 31062 Toulouse, France.

${ }^{b}$ University of Hamburg, Center of Earth System Research and Sustainability (CEN), Institute of Marine Ecosystem and Fishery Science (IMF), Große Elbstraße 133, 22767 Hamburg, Germany.

' Institut de Recherche sur les Forêts, Université du Québec en Abitibi-Témiscamingue, 445 Boul. de l'Université, Rouyn-Noranda, QC J9X 5E4, Canada.

${ }^{\mathrm{d} H u m a n}$ Evolutionary Genetics Unit, Institut Pasteur, UMR 2000, CNRS, Paris 75015, France.

eUniversité Paris Diderot, Sorbonne Paris Cité, Paris 75013, France.

${ }^{1}$ Corresponding author. Email: robin.aguilee@univ-tlse3.fr

\section{ORCIDs:}

RA: 0000-0002-3388-9921

FP: 0000-0002-7062-4957

MS: 0000-0003-0356-8681

JC: 0000-0002-2189-8767

CT: 0000-0002-8586-1234 


\section{Abstract}

Aim: Accounting for geo-environmental dynamics is crucial to understand community assembly across islands. Whittaker et al. (2008)'s General Dynamic Model aims towards this goal. Yet it does not explicitly consider that most islands belong to archipelagos. We examined how island biodiversity dynamics are influenced by the interaction of eco-evolutionary processes acting at the archipelago level with each island's geo-environmental dynamics.

Location: Hypothetical archipelagos.

Taxon: Any.

Methods: We used an individual-based model, ecologically neutral within the archipelago. Several islands emerge in succession with a typical volcanic ontogeny. We considered both mainland and inter-island dispersal. Geographically isolated lineages diverged over time, possibly speciating.

Results: We found diversity to be at a dynamic equilibrium. In an archipelago, islands hosted more diversity and more endemic species, at both island and archipelago levels, than an equivalently-sized single isolated island. This was due to an "archipelago effect": inter-island dispersal increased within-island diversity through species occurrence on multiple islands; species may undergo anagenetic changes on the colonised islands, eventually speciating, thereby increasing archipelago diversity. Biodiversity dynamics of different islands may differ even on islands with identical geo-environmental dynamics because the archipelago effect varied over time and affected each island differently ("history effect"). By accounting for these effects, we predicted detectable deviations from the General Dynamic Model predictions which are largest for remote archipelagos, with islands located close together, and with intermediate time of island emergence. In linear stepping-stone archipelagos, we predicted higher diversity on centrally located islands.

Main conclusions: Our results demonstrate that analyses of insular biodiversity data would greatly benefit from explicitly accounting for both archipelago and history effects. We suggest incorporating variables characterising the spatio-temporal structure of the whole archipelago. We discuss possible difficulties in distinguishing between the archipelago effect and equilibrium diversity dynamics.

Keywords: biodiversity dynamics, equilibrium, General Dynamic Model, island biogeography, island ontogeny, mechanistic model, speciation, species richness, volcanic island. 


\section{Introduction}

Understanding the processes driving the buildup of species communities in simple systems such as marine islands remains a central theme in ecology, biogeography, and evolutionary biology (Losos and Ricklefs, 2009; Warren et al., 2015; Whittaker et al., 2017). In oceanic islands that were never connected to any continent, the accumulation of species diversity depends on how the processes of colonisation, speciation, and extinction are shaped by the geographical context (MacArthur and Wilson, 1963, 1967; Valente et al., 2020). Yet, discrepancies are often observed between straight island biogeographical predictions and patterns of diversity across islands, mainly because geographical settings change over time (Ricklefs, 2004; Whittaker and Fernández-Palacios, 2007; Warren et al., 2010).

Many recent studies in island biogeography have used the General Dynamic Model (hereafter, GDM; Whittaker et al., 2008) framework to address this issue in the case of volcanic oceanic islands. The GDM is a conceptual model based on the equilibrium theory of MacArthur and Wilson $(1963,1967)$ which postulates that island diversity results from a diversity-dependent dynamic balance between immigration, speciation, and extinction, where these eco-evolutionary processes are determined by the size and isolation of the island. One important addition of the GDM to MacArthur and Wilson's original model was the incorporation of spatio-temporal changes in island characteristics to account for continuous changes in geographical settings (Whittaker et al., 2008): the GDM postulates that the surface area, altitude and topographical complexity of an island changes over the island's lifetime. The resulting main prediction is that species richness and the number of endemic species varies as a hump-shaped function of time (Whittaker et al., 2008; Borregaard et al., 2017).

The GDM was mathematically (Chen et al., 2011; Valente et al., 2014) and computationally (Borregaard et al., 2016; Cabral et al., 2019) assessed, and adapted to different types of islands (e.g. subduction-based arc islands and continental fragments; Borregaard et al., 2016). Its main prediction was empirically evaluated using data from many different archipelagos and taxa (reviewed in Borregaard et al., 2017), mostly using statistical models which assess the relationship between diversity and two explanatory variables, the size and the age of the island. The main prediction of the GDM appears to be reasonably supported by empirical testing, although this is not always the case (Steinbauer et al., 2013; Borregaard et al., 2017).

One possible reason for this partial mismatch is that the rate of landscape changes may outpace that of biological processes, causing non-equilibrium dynamics (Warren et al., 2015). The discovery of island biotas that are out of equilibrium have accumulated, encouraging many authors to call for the development of a non-equilibrium theory of island biogeography (Heaney, 2000; Lomolino, 2000; Whittaker, 2000; Heaney et al., 2013; Warren et al., 2015). However, recent studies at large geographical scales concluded that island biodiversity is generally at equilibrium (Valente et al., 2017, 2020).

Another possible reason for the partial mismatch between the GDM predictions and empirical testing is that many islands are located in archipelagos, 
a feature which is not explicitly accounted for in the GDM. An archipelago is defined as a group of islands which exchange biological information among themselves (migrants) more often than they do with other landmasses (Whittaker et al., 2018). In an archipelago, inter-island dispersal may increase diversity at the island scale: any species may occur on several islands. Inter-island dispersal may also alter endemic status, with single-island endemic species becoming multiple-island endemic species. Also, the contribution of demographics due to inter-island dispersal can reduce local extinction rates (rescue effect; Brown and Kodric-Brown, 1977). Colonist lineages which have dispersed to different islands within an archipelago can diverge and eventually become distinct species (within-archipelago speciation). This process may be hampered, and even prevented, when inter-island dispersal is so frequent that gene flow homogenises populations (e.g. Casquet et al., 2015). Finally, some islands may be the first to be colonised, thus serving as a stepping stone that favours the colonisation of other islands in the archipelago (Shaw and Gillespie, 2016). Because these eco-evolutionary processes act at the archipelago scale, the archipelago has been increasingly recognised as the relevant scale to study island biodiversity data (Triantis et al., 2015, 2016; Borregaard et al., 2017; Whittaker et al., 2018; Ali and Meiri, 2019). In an attempt to account for archipelago structures, statistical fits to the GDM now often use linear mixed-effects models with archipelago identity as random variable (Bunnefeld and Phillimore, 2012; Borregaard et al., 2017). However, we still do not fully understand how the mechanisms that operate at the scale of an archipelago affect biodiversity dynamics on islands.

In a theoretical paper, Gascuel et al. (2016) investigated the consequences of archipelago-specific eco-evolutionary processes on species richness. Using an individual-based model, these authors showed that greater total diversity is to be expected in an archipelago than on a single island of the same size as the whole archipelago, a phenomenon that was linked to the proliferation of anagenetic species on different islands. In another theoretical study, Jõks and Pärtel (2018) explored the effect of the relative position of islands in an archipelago and showed that the spatial configuration of the archipelago does matter in explaining the composition of species communities. A few empirical analyses also explicitly accounted for the eco-evolutionary processes at archipelago scale, overall providing good support for theoretical predictions (Cabral et al., 2014; Triantis et al., 2015; Ali and Meiri, 2019). However, one strong limitation of these theoretical and empirical studies is that they all consider archipelagos as static entities, i.e., they ignore the island geoenvironmental dynamics incorporated in the GDM for a single isolated island. A step forward was taken by Jõks et al. (2021) who showed that archipelago ontogeny may leave signatures on current biodiversity patterns. Yet, Jõks et al. (2021) restricted their modelling of archipelago-specific processes to the situation where inter-island dispersal has no evolutionary consequences, i.e. it neither triggers anagenetic divergence nor delays speciation. The consequences of the intricate and temporally variable eco-evolutionary processes acting at the archipelago scale on biodiversity dynamics therefore remain to be elucidated.

In this study, we postulate that considering both the archipelago-specific eco-evolutionary processes and the whole archipelago ontogeny should lead 
to biodiversity dynamics that are distinct from those predicted by previous studies. Specifically, we hypothesise that:

1. Diversity dynamics lags behind the eco-evolutionary equilibrium.

2. Diversity dynamics on each island deviates from the GDM prediction, with overall higher diversity, because the archipelago processes trigger speciation.

3. Diversity dynamics differ on each island in the archipelago because of continuous alterations of the connectivity patterns as islands emerge, change size and disappear.

We expect that the spatio-temporal structure of the archipelago influences the magnitude of the deviations from GDM predictions. Deviations should be larger when the archipelago-specific eco-evolutionary processes are stronger than other processes that are not specific to the archipelago scale. Therefore, we hypothesise that:

4. Deviations from the GDM predictions are larger for archipelagos with islands located geographically closer to each other (4a), that emerge more rapidly one after the other (4b) and are located farthest from the mainland $(4 \mathrm{c})$. In the case where successively emerging islands are also the geographically closest islands, like in linear stepping stone archipelagos, we predict larger deviations from the GDM for the closest (in space and time) islands (4d).

We used an individual-based model to test these hypotheses. The model explicitly accounts for the whole life cycle of a volcanic oceanic archipelago by considering the successive emergence of multiple islands, as encountered for example in oceanic archipelagos associated with a mantle-plume hotspot, and by considering a typical volcanic ontogeny for each island. It explicitly includes inter-island dispersal, its temporal variations, and its evolutionary consequences. We analysed the dynamics of species richness at island and archipelago scales, as well as the dynamics of endemic species, tracking the eco-evolutionary processes that cause the observed diversity dynamics.

\section{Methods}

We first designed a baseline model of community dynamics in an evolving archipelago setting. The baseline model is built on five guiding assumptions, illustrated in Fig. 1: (i) several islands appear and disappear during the lifetime of the archipelago; (ii) each island satisfies the premises of the GDM, in particular the carrying capacity of each island is seen as a hump-shaped function of time; (iii) inter-island dispersal depends on the size of the sources and of the destination, and therefore varies over time in a possibly non-monotonic way; (iv) colonisation of an island by a species that was previously absent on that island triggers divergence of the colonist lineage from the source population; and (v) speciation is protracted (Rosindell and Phillimore, 2011) and delayed by gene flow. We detail the assumptions of the model below. The 
corresponding technical details are provided in Appendix S1 in Supporting Information. After analysing the baseline model, we explore more complex geo-environmental situations.

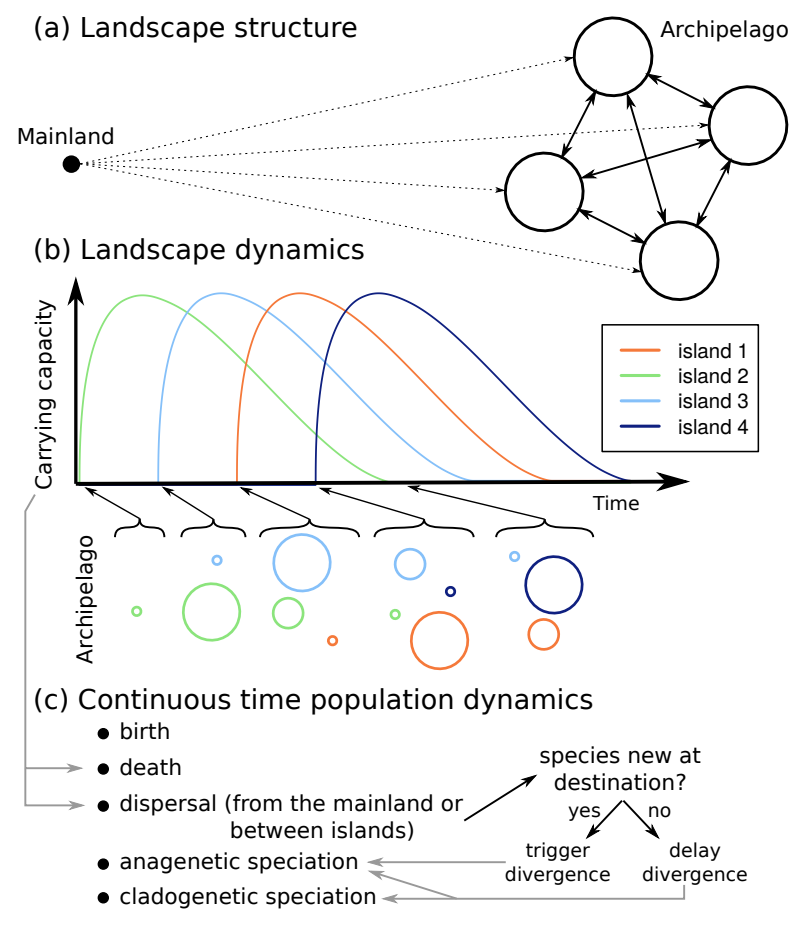

Figure 1: Main assumptions of the baseline model. Panel (a) depicts the landscape structure. Arrows with the same line style (solid or dotted) indicate a similar dispersal rate if the islands are of similar size (i.e. arrows with the same line style indicate identical geographical distances even though the arrows are of different lengths). Panel (b) shows the landscape dynamics, i.e., the island carrying capacity over time, with an illustration of the archipelago structure sampled at the five times indicated by the arrows. Panel (c) outlines the population dynamics. Most processes influence each other through feedback (see explanation in the text); gray arrows link processes which have a direct influence on other processes.

\subsection{Landscape dynamics}

Landscape dynamics consists of the ontogeny of $n$ islands appearing at different times during the archipelago's life cycle. In the baseline model, islands appear successively with a time interval denoted $t_{\text {inter }}$ between the emergence of each island (see Table S2.1 in Appendix S2 for a summary of the notation and default parameter values). Each island undergoes changes in its geo-environmental characteristics resulting in a varying carrying capacity expressed as the number of individuals, $K(t)$, which is a hump-shaped function of time. The carrying capacity function follows the life cycle of a volcanic oceanic island: it reaches its maximum value $K_{\max }$ at the beginning of its life, that is, in a proportion $p_{\max }=0.2$ of its lifetime $t_{\text {life }}$. In the baseline model, the carrying capacity function parameters have the same value for all islands, i.e., the geo-environmental dynamics of all the islands are the same. 
The distance between emerged islands, $d_{\mathrm{i}}$, is held constant as is the distance to the mainland, $d_{c}$. This implies that distances between the landmasses (i.e., the landmass of all the islands and that of the mainland) are large compared to variations in the size of the islands. In the baseline model, the distance between islands is identical for all pairs of islands, and the distance to the mainland is the same for all the islands. This geographical configuration is physically impossible in an archipelago containing more than two islands. We nevertheless use it, with $n \geq 2$ islands, since it avoids any mixed effect due to a specific spatial structure and thus provides a useful virtual configuration to investigate intra-archipelago processes. In the second step, we analyse realistic archipelago structures (see Section 2.4).

We do not take the internal geography of the mainland into account as it is assumed to be a point with all individuals at the same position. This assumption is especially realistic for remote archipelagos. We simulate the mainland species composition as a large neutral metacommunity at equilibrium with a species-abundance distribution consistent with predictions of the unified neutral theory of biodiversity (Hubbell, 2001), i.e. a log-series distribution with a few abundant species and many rare species. This distribution is generated stochastically at the beginning of each simulation with a metacommunity of constant size $J$ and a constant fundamental biodiversity number $\theta$. We assume that the mainland community remains unchanged throughout the lifetime of the archipelago.

\subsection{Population dynamics}

The model is stochastic, individual-based, and ecologically neutral within the archipelago. Generations overlap. Throughout this article, an "individual" is defined as a propagule in the sense of the smallest number of individuals comprising a population that can persist. Population dynamics consists of five possible events on each island: birth of an individual, death of an individual, cladogenetic speciation, anagenetic speciation and immigration of an individual (from the mainland or from an island).

Birth occurs at constant rate $\beta$. New-born individuals inherit the species identity of their parent. Death is density-dependent at the scale of the island.

We model cladogenesis following Rosindell and Phillimore (2011): each individual initiates a differentiating lineage at constant rate $\sigma$. We model anagenesis following Gascuel et al. (2016): each migrant (from the mainland or from an island) initiates a differentiating lineage if the migrant species is new on the destination island. Note that in the case of anagenesis following interisland dispersal, it can be considered as cladogenesis from the perspective of the archipelago. Speciation (cladogenesis and anagenesis) is protracted (i.e. it takes time; Rosindell and Phillimore, 2011; Gascuel et al., 2016): it is completed after a constant minimum time $\tau$ plus a variable delay that extends with (i) increasing gene flow from the same species, and with (ii) decreasing population size of the differentiating lineage, which accounts for the fact that introgression from one individual has more genetic consequences in small populations than in large populations.

Dispersal occurs from the mainland to the islands (but not from the islands 
to the mainland, i.e., there is no boomerang effect), and between islands within the archipelago. Each individual emigrates at a constant rate $\mu$. The number of emigration events is thus proportional to the size of the source populations. The probability of successful immigration increases with the size of the destination island (estimated by its carrying capacity $K$ ) because, for dispersal to be successful, a migrant must disperse in a direction that enables it to reach its destination, which is more likely if the destination is large and located close by (target area effect; Gilpin and Diamond, 1976). At the archipelago scale, since the sizes of all the islands (as source or destination) are functions of time, dispersal (emigration and immigration) is time-dependent: successful dispersal varies with the ontogeny history of the whole archipelago and may change in a complex way.

The success of dispersal decreases with increasing distance between the source and the target. We use the power-law dispersal kernel from Clark et al. (1999), which also allows for long-distance dispersal events. We denote $c$ the parameter controlling the dispersal ability of the species. We account for the widespread loss of dispersal abilities in island species (Waters et al., 2020) by assuming that individuals on islands have a lower emigration rate $\mu$ and a lower dispersal ability $c$ than individuals on the mainland (Table S2.1). We only use this assumption for the purpose of illustration; there are empirical cases where, on the contrary, island species have better dispersal abilities (Whittaker and Fernández-Palacios, 2007). Such cases are indirectly visible in our analysis of archipelagos with different structures because in our model, increasing the dispersal rate and dispersal ability of island species is equivalent to reducing the geographical distance between islands: it increases effective dispersal between islands.

\section{$2.3 \quad$ Null models}

We address the consequences of eco-evolutionary processes at the scale of the archipelago for within-island diversity by comparing the results obtained for each island with a "single isolated island" null model. This null model corresponds to the GDM. The dynamics of the carrying capacity of the single isolated island resembles that of any island in the baseline model.

We extract the consequences of archipelago structure for diversity at the archipelago scale by comparing the results of the baseline model with an "island archipelago-equivalent" null model. This null model corresponds to a single isolated island whose carrying capacity dynamics is the same as that of the whole corresponding archipelago (i.e., the sum of $K$ over all islands).

We check whether biodiversity is at equilibrium by comparing the results of the baseline model with a "static archipelago" null model. This null model computes the eco-evolutionary equilibrium diversity at each time. To this end, we sample the archipelago configurations from the baseline model every $10^{4}$ generations. For each successive archipelago configuration, we compute the equilibrium by running a sub-model routine with a static landscape (i.e., islands of constant size) corresponding to the sampled configuration until species richness reaches equilibrium. We then plot the equilibrium species richness as a function of time of the main model routine, i.e., as a function of the successive 
archipelago configurations. We also compute the static models using the same approach for the "single isolated island" and "island archipelago-equivalent" null models.

\subsection{Correlated spatial position and emergence time}

In addition to the baseline model, we analyse two spatial structures in which the spatial position of the islands and their emergence time are correlated, as typically encountered in archipelagos formed by an oceanic plate moving above a mantle-plume hotspot. First, we consider a linear stepping stone with new islands emerging in the extension of the stepping stone and with all islands at the same distance from the mainland, i.e. an archipelago parallel to the mainland, or so far from the mainland that all the islands can be considered as being at the same distance from the mainland, such as the Hawaiian archipelago. Second, we analyse a linear stepping stone perpendicular to the mainland in which the first emerging island is the one closest to the mainland, a situation found for example in the Canary Islands archipelago.

\subsection{Numerical simulation methods}

We simulate the model forward in time using Gillespie (1977)'s algorithm in continuous time. We measure time in generations. The simulation program is embedded in a script written in the $\mathrm{R}$ language ( $\mathrm{R}$ Core Team, 2017) to allow easy replication and extension.

For each set of parameter values, we run 25 replicate simulations. For each output, we show the mean value of all the replicate simulations.

Initially the archipelago is empty of individuals. A mainland speciesabundance distribution is generated for each replicate simulation.

\subsection{Model analysis methods}

The chosen default parameter values (Table S2.1) represent a weakly dispersing clade, such as snails and flowering plants, whose populations can differentiate at small spatial scales (Kisel and Barraclough, 2010).

We monitor several outputs that characterise the state and evolution of the island populations: species richness, proportion of endemic species, proportion of single- and multiple-island endemics among all species, dispersal rates to a focal island from the mainland and from the other islands of the archipelago, rates of anagenesis initiation (after dispersal events from the mainland and from the other islands) which reflect colonisation rates (i.e. dispersal rates of species previously absent at the destination), the rate of cladogenesis initiation, rates of speciation from each origin (cladogenesis, and anagenesis after dispersal from the mainland and from an other island), duration of speciation (value averaged over the endemic species currently present on each island), and the rate of species extinction. We measure the outputs for each individual island and at the scale of the archipelago. To better visualise the results, in some figures, we plot the relative change in local diversity for the archipelago model compared to the single isolated island model, which is computed as: (species 
richness in the archipelago model - species richness in the single isolated island model) / species richness in the single isolated island model.

We vary all parameters of the model to evaluate their influence on community dynamics. Results concerning inter-island distance $\left(d_{\mathrm{i}}\right)$, time interval between island emergence $\left(t_{\text {inter }}\right)$, and distance to the mainland $\left(d_{\mathrm{c}}\right)$ are given in the main text, the results on other parameters are given Appendix S3.

\section{Results}

\subsection{Equilibrium}

Comparison of the baseline model with the null static archipelago model (i.e. a model computing diversity at the eco-evolutionary equilibrium at each time) shows that species richness is at equilibrium at all times (Fig. 2b,c). Although we cannot prove that non-equilibrium dynamics are impossible in our model, we never observed any despite exploring a wide parameter space (Appendix S3). Hypothesis 1 is thus not confirmed. Additionally, species richness on each island peaked later than carrying capacity except for the last island to emerge (Fig. 2a,c).

\subsection{Deviations from the GDM: the archipelago effect}

In the baseline model, we found that as soon as there was more than one island in the archipelago, species richness at the archipelago scale (hereafter, total diversity) became higher than in the corresponding island archipelagoequivalent null model (i.e., a single island with the archipelago's carrying capacity; Fig. 2b). Similarly, within-island species richness (hereafter, local diversity) on any island in the archipelago became higher than in the corresponding single isolated island null model (i.e., model corresponding to the GDM; Fig. 2c). As long as several islands coexisted in the archipelago, the proportion of endemic species (single- and multiple-island endemics) on any island was higher than in the single isolated island model (Fig. 2d; see also Fig. S2.3 in Appendix S2).

Our analysis made it possible to track which of the eco-evolutionary processes included in the model causes higher diversity than expected under the GDM. We call this set of eco-evolutionary processes the "archipelago effect", and explain it is as follows: within an archipelago, the occurrence of interisland dispersal (Fig. 3a) had two major consequences for diversity. First, some species occurred on several islands, which increased local diversity (but not total diversity). This effect can be seen on the proportion of multiple-island endemics (among all species) which jumped each time a new island appeared while the proportion of single-island endemics dropped (Fig. 3b). Not all interisland dispersal events of locally new species led to a persistent increase of local diversity, because the arrival of migrants also amplified the rate of local species extinction (Fig. 3c). Second, inter-island colonisation (i.e. dispersal of locally new species) promoted differentiation of anagenetic lineages between islands (Fig. 3d), some of which led to speciation events (Fig. 3e,f) with a delay due to inter-island dispersal (gene flow) offsetting differentiation (Fig. 3g). These 

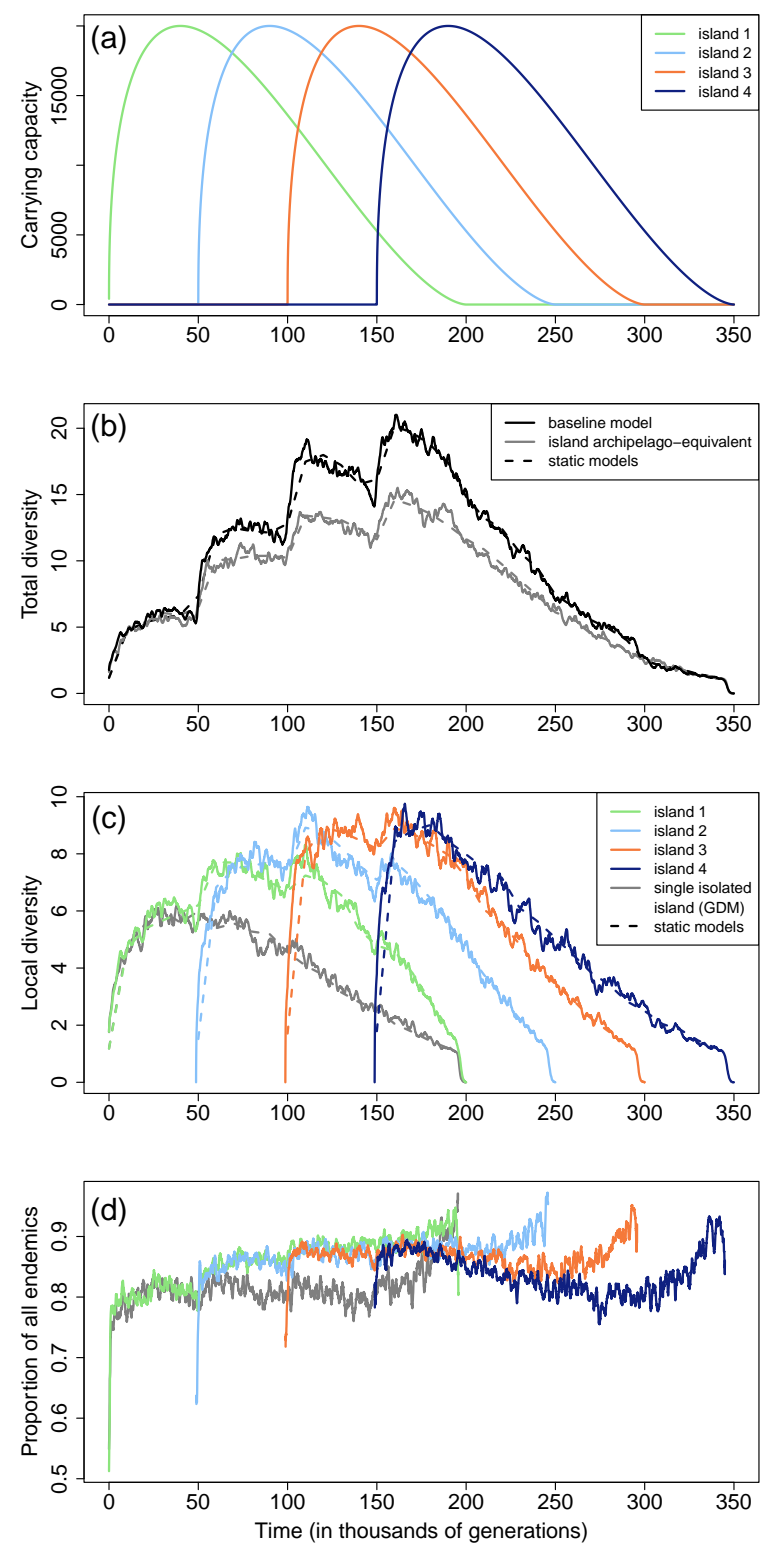

Figure 2: Mean dynamics over 25 replicate simulations of carrying capacity (a), diversity at the scale of the archipelago (b), diversity on each island (c), and the proportion of all endemic species (single- and multiple-island endemics) on each island (d) in the baseline model and in the null models. Islands 1 to 4 correspond to the four islands in the baseline model. Default parameter values (Table S2.1).

additional speciation events, which can only occur in an archipelago, increased total diversity, and a fortiori local diversity, as well as endemism (Fig. 2b-d). The other eco-evolutionary rates that possibly contribute to variations in diversity (dispersal from the mainland, anagenesis following mainland dispersal, and cladogenesis) were identical for each island of the archipelago and for a single isolated island (Fig. S2.4), and therefore did not contribute to the observed deviations of the diversity dynamics from those expected in the GDM. The efficiency of the archipelago effect in increasing diversity was determined by the abundance of inter-island migrants and by the dissimilarity of the com- 
munity composition of this migrant pool compared to the local community. Everything else being equal, when an island received a substantial number of inter-island migrants and/or the migrant community was highly dissimilar from the local community, inter-island migrants were more likely to be new on the destination island, which increased local diversity, and also started new differentiating lineages, eventually leading to speciation, thereby increasing total diversity.
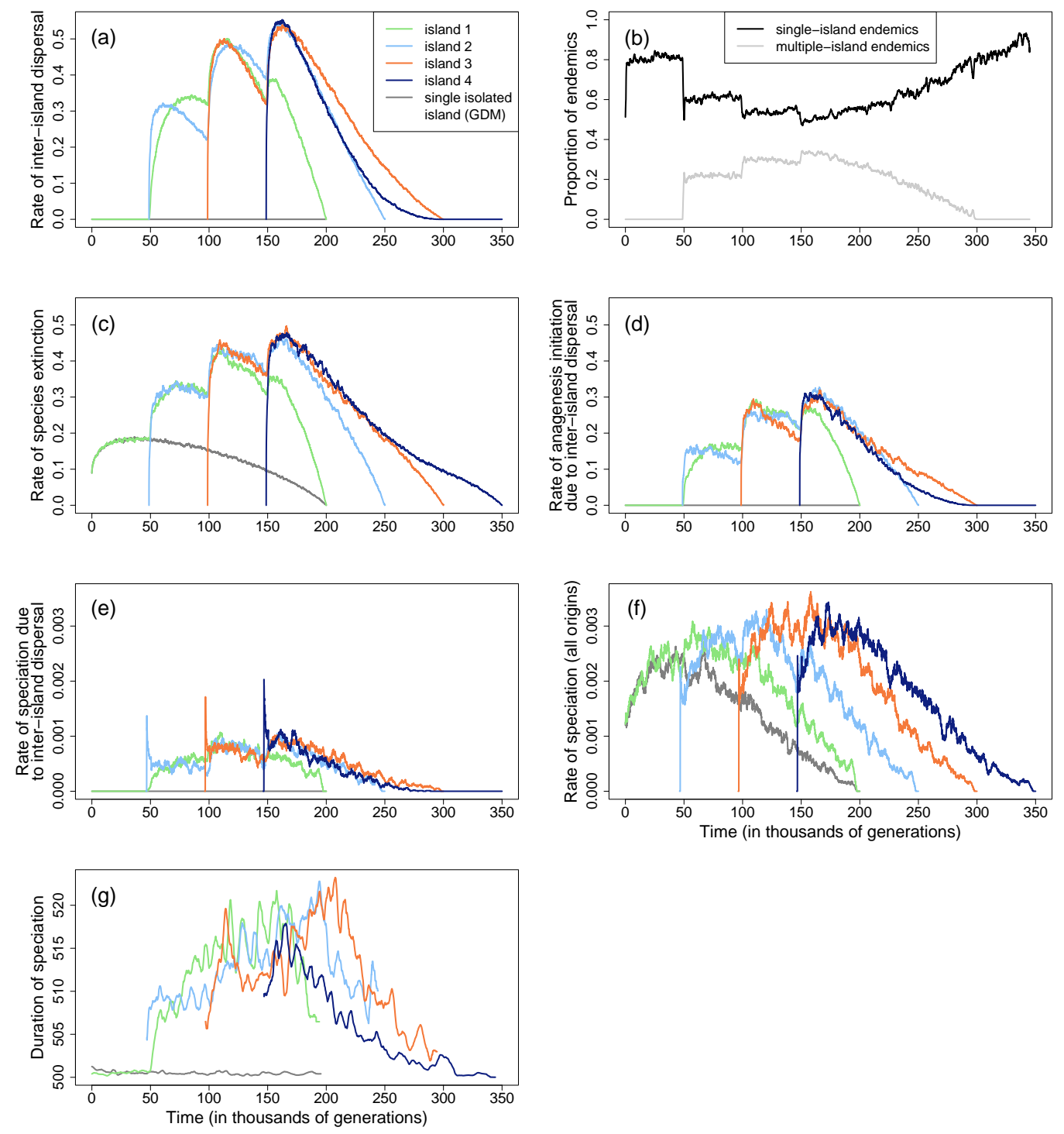

Figure 3: Mean dynamics over 25 replicate simulations of different outputs that characterise the state and evolution of the population (see Section 2.6) in the baseline model and in the single isolated island model. The archipelago effect corresponds to the difference between each island's output and the same output for a single isolated island assumed to emerge at the same time. The proportions of single- and multiple-island endemics (panel (b)) are computed among all species at the archipelago scale. The rate of anagenesis initiation due to inter-island dispersal (panel (d)) reflects the inter-island dispersal rate of locally new species. Default parameter values (Table S2.1). Several islands coexist between times 50 and 300 (in thousands of generations; Fig. 2a). 
To sum up, we demonstrate that (i) total and local diversity as well as endemism are higher than expected under the GDM, and that (ii) this is caused by the archipelago processes triggering speciation, i.e. hypothesis 2 is confirmed.

\subsection{Differences between islands: the history effect}

In the baseline model, total and local diversity were generally hump-shaped functions of time, but included significant deviations from a smooth curve when new islands emerged (Fig. 2b,c). In addition, despite having identical geoenvironmental dynamics (Fig. 2a), islands within the archipelago had different biodiversity dynamics (Fig. 2c): they reached different maximum levels of diversity, did not reach maximum at the same stage of their life cycle, diversity remained maximum for different length of time, and biodiversity dynamics followed hump-shaped functions of time with different frequencies of sudden jumps. The dynamics of the proportion of endemic species also differed on each island (Fig. 2d and Fig. S2.3).

We tracked which eco-evolutionary processes caused such differences between islands. We call this set of processes the "history effect". It has two components. First, dependence on time results from the geo-environmental dynamics of each island (Fig. 2a). This corresponds to the GDM and explains why local species richness was a roughly hump-shaped function of time (Fig. 2c). Second, the intensity of the archipelago effect varied over the life cycle of the archipelago (Fig. 3). The abundance of inter-island migrants received and the dissimilarity of their community from the local community indeed varied over time in our simulations. For example, under the archipelago ontogeny of the baseline model, islands 2 and 3 were those which received the most inter-island migrants (Fig. 3a), because the cumulative size of their neighbour islands was high throughout their lifetime (Fig. S2.5). The dissimilarity between the inter-island migrant community and the local community was also the highest during the lifetimes of islands 2 and 3 (Fig. 3d). These two islands therefore displayed the strongest and longest lasting archipelago effect, and the maximum local diversity was both the highest and the most persistent on these two islands (Fig. 2c). Island 1 experienced a stronger archipelago effect leading to local diversity which differed more from that expected with the GDM in the later part of its life cycle. The reverse was true for island 4 which experienced a higher archipelago effect at emergence. Finally, in the baseline model, islands which appeared earlier in the life cycle of the archipelago experienced the emergence of more neighbour islands, and therefore more variations in the intensity of the archipelago effect (e.g. island 1 vs 4 on Fig. 3a,d). This led local diversity dynamics to follow a hump-shaped function of time with more frequent sudden jumps (island 1 vs 4 on Fig. 2c).

To sum up, we demonstrate that (i) diversity dynamics differ on each island in the archipelago, and that (ii) this is caused by continuous alterations of the connectivity patterns over the archipelago life cycle, i.e. hypothesis 3 is confirmed. 


\subsection{Archipelagos with different structures}

\subsubsection{Inter-island distance}

When islands were geographically very close to one another, we found that the archipelago effect and its variations over time led to marked deviations from GDM predictions (Fig. 4a), i.e., hypothesis 4a is confirmed. In this geographical context, islands exchanged many migrants, which led to a high proportion of species occurring on multiple islands and to a high rate of speciation, despite the fact that speciation was significantly delayed by inter-island dispersal (Fig. S2.6). Consequently, local species richness was found to be very high compared to that on a single isolated island (Fig. 4a). Because the rates of inter-island dispersal and of speciation varied considerably over time when islands were located close to one another (i.e., there was a strong history effect; Fig. S2.6), local diversity dynamics followed hump-shaped functions of island age with very clear sudden jumps, and differed markedly between islands (Fig. 4a).

\subsubsection{Time interval between the successive emergence of islands}

When islands emerged rapidly one after the other (i.e., large temporal overlap between the existence of the islands), we found a striking increase in diversity compared to the GDM prediction (Fig. 4b), which was due to a strong archipelago effect (Fig. S2.7). However, we also observed almost no difference in diversity dynamics among islands (Fig. 4b), because the history effect was identical for all islands (Fig. S2.7). This could make the deviations from the GDM predictions difficult to detect despite their magnitude. Hypothesis $4 \mathrm{~b}$ is thus only partially confirmed.

\subsubsection{Distance to the mainland}

When the archipelago was located farther from the mainland than in the baseline model, we found that the increase in diversity compared to the GDM prediction was larger (Fig. 4c), i.e. hypothesis 4c is confirmed. The archipelago effect per se did not vary with the distance from the mainland (Fig. S2.8), but the importance of the archipelago effect compared to mainland dispersal (and its evolutionary consequences) was higher when the archipelago was located farther from the mainland. Dispersal from the mainland was indeed lower in this case, which resulted in fewer continental species, thus in increased proportion of endemic species (Fig. S2.8). In turn, this led to fewer lineages diverging from the mainland eventually leading to speciation, and hence to a lower contribution of mainland species to diversity dynamics.

\subsubsection{Correlated spatial position and emergence time}

In the case of an archipelago shaped like a linear stepping stone parallel to the mainland (or so far from the mainland that all islands can be considered as being at the same distance from the mainland), the archipelago effect was concentrated among the islands located closest together (geographically and temporally; Fig. S2.9), resulting in larger deviations from the GDM prediction 

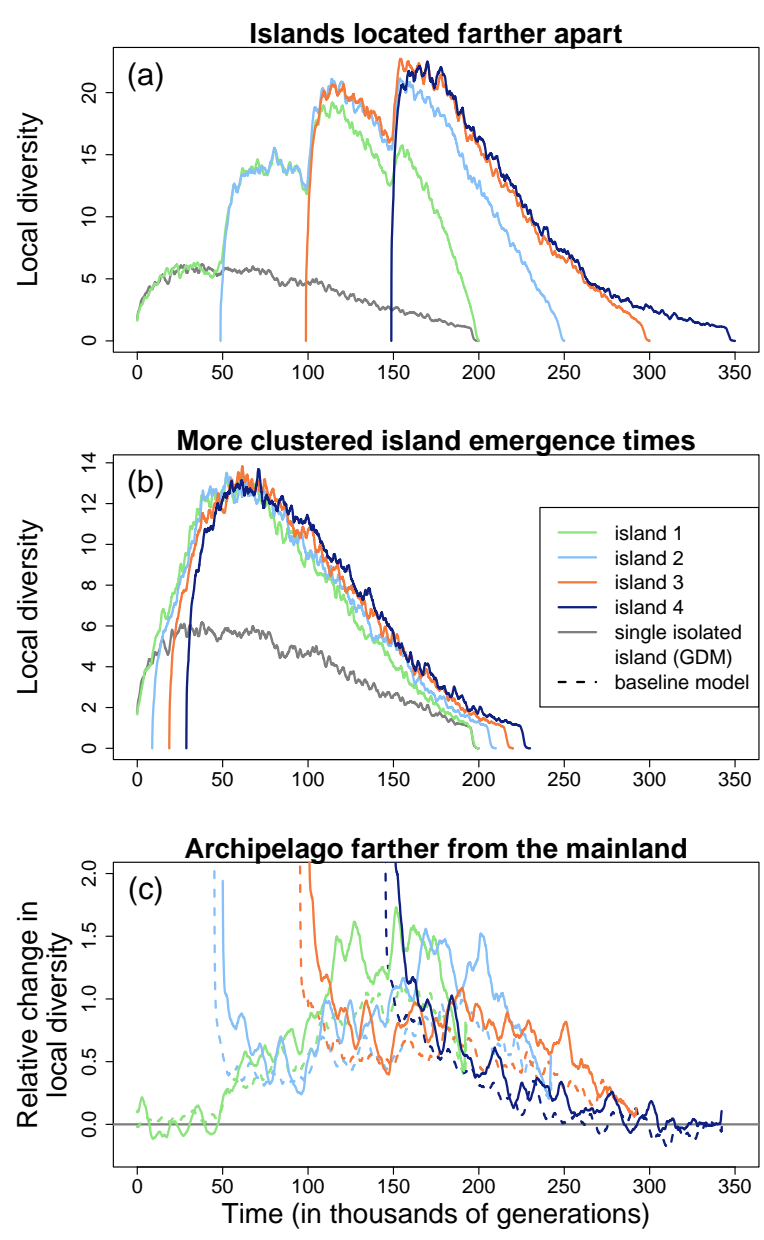

Figure 4: Mean dynamics over 25 replicate simulations of local diversity in an archipelago where islands are located farther from each other than in the baseline model (a; inter-island distance $d_{\mathrm{i}}=10$ ), and of local diversity in an archipelago where islands emerge successively faster than in the baseline model (b; time interval between island emergence $t_{\text {inter }}=10^{4}$ ). In panel $(\mathrm{c})$, the archipelago is located farther from the mainland than in the baseline model (distance to the mainland $d_{\mathrm{c}}=250$ ). To better visualise the results, this panel shows the relative change in local diversity for the archipelago model compared to the single isolated island model, computed as: (species richness in the archipelago model - species richness in the single isolated island model) / species richness in the single isolated island model. The distance to the mainland is the same in the single isolated island model and in the corresponding archipelago model. In all panels, the other parameters are set to default values (Table S2.1). All panels show the results for the archipelago model; panels (a) and (b) also include a line for the single isolated island model; panel (c) also includes the results of the baseline model for comparison.

for centrally placed islands (islands 2 and 3; Fig. 5a). Hypothesis 4 d is therefore confirmed for such archipelagos. Larger deviations from the GDM prediction resulted in higher local diversity in centrally located islands than in islands at the margins of the archipelagos (Fig. S2.9). Because the latter islands received fewer inter-island migrants than the former, the archipelago effect was weaker and their species richness was consequently also lower (Fig. S2.9), even when 

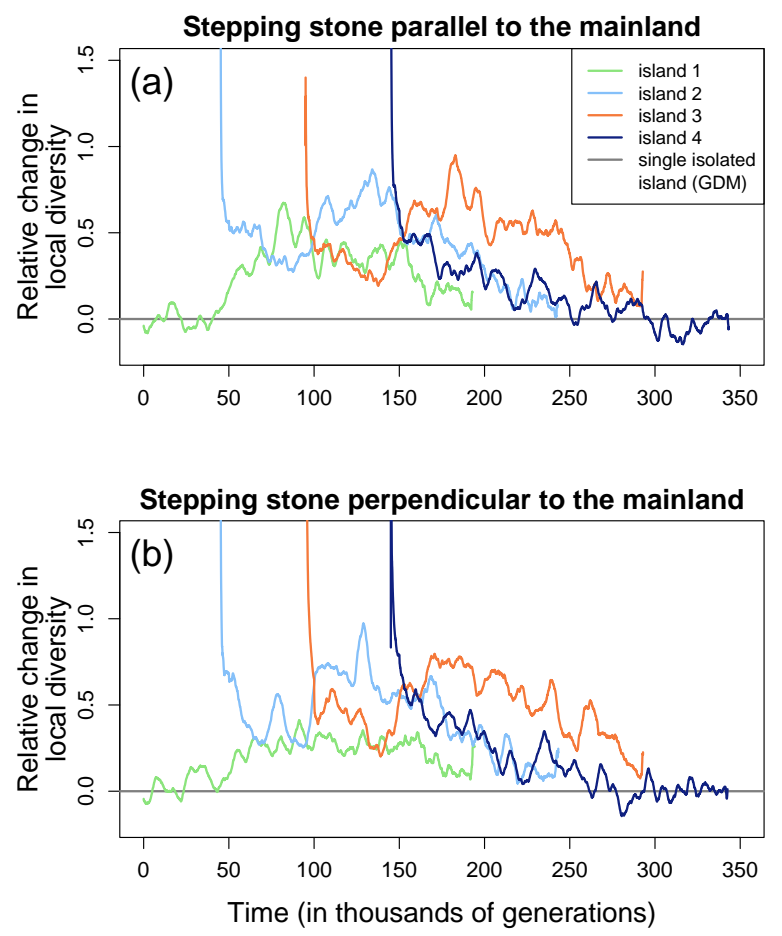

Figure 5: Relative change (mean over 25 replicate simulations) in local diversity for the archipelago model compared to the single isolated island model in an archipelago shaped like (a) a linear stepping stone parallel to the mainland or so far from the mainland that all islands can be considered as being at the same distance from the mainland, e.g. the Hawaiian archipelago, or like (b) a linear stepping stone perpendicular to the mainland where the first emerging island is closest to the mainland, e.g. the Canary Islands. We plot here the relative change in diversity to better visualise the differences with the GDM predictions. It is computed as: (species richness in the archipelago model species richness in the single isolated island model) / species richness in the single isolated island model. In panel (b), the average distance between the islands and the mainland is $d_{\mathrm{c}}=150$ like in the baseline model, which means that the distance between the first (respectively, last) emerging island and the mainland is less (respectively, more) than 150 . The relative change in diversity is computed for each island with the single isolated island model at the same distance to the mainland as for the island considered. In both panels, the distance between neighbour islands is $d_{\mathrm{i}}=20$ like in the baseline model, which means that the distance between non-neighbour islands is greater than 20. The other parameters are set to default values (Table S2.1).

the islands emerged simultaneously (Fig. S2.10).

In the case of a linear stepping stone archipelago perpendicular to the mainland, with the first emerging island the closest to the mainland, we also observed larger deviations from the GDM prediction for centrally located islands (Fig. 5b), i.e. hypothesis $4 \mathrm{~d}$ is also confirmed for this kind of archipelago. The influence of diversity originating from the mainland is the strongest for the first island to emerge, because it is also the closest to the mainland. As a consequence, deviations from the GDM prediction for this island were smaller 
in a stepping stone perpendicular to the mainland than in a stepping stone parallel to the mainland (Fig. 5).

\section{Discussion}

The General Dynamic Model (GDM; Whittaker et al., 2008) predicts that species richness on a volcanic oceanic island will be a hump-shaped function of time resulting from the geo-environmental dynamics of the island. Using a theoretical approach, we show (i) that, for individual islands within an archipelago, significant deviations from this prediction are to be expected, with higher diversity than that predicted for a single isolated island, and (ii) that, even if the geo-environmental dynamics of islands within an archipelago are identical, their biodiversity dynamics may be strikingly different (Fig. 2). We demonstrate that these differences arise from of an "archipelago effect" caused by inter-island dispersal and its evolutionary consequences, combined with a "history effect" which makes the archipelago effect vary over time (Fig. 3). These results are entirely consistent with the view that the archipelago may be, at least in some cases, a more suitable scale than the island to understand island biodiversity data (Triantis et al., 2015, 2016; Borregaard et al., 2017; Whittaker et al., 2018; Ali and Meiri, 2019). Below, we argue that both archipelago and history effects should be explicitly taken into account to understand insular biodiversity patterns, and we stress under which circumstances it is most valuable to do so.

The archipelago effect highlighted in our model results from (i) inter-island dispersal which increases local diversity through species occurrence on multiple islands, and (ii) species undergoing anagenetic changes within islands they have colonised, eventually leading to speciation, thereby increasing diversity at the archipelago scale. An analogous effect was described by Haydon et al. (1993) in the context of an archipelago with a vicariant history (an initial island continuously splitting itself into smaller units while the size of the archipelago remained constant), and also by Gascuel et al. (2016) who modelled an archipelago with islands that remained stable over time. The archipelago modelled here is fully dynamic in the sense that we explicitly consider the geo-environmental dynamics of each individual island. Consequently, the archipelago effect varies over time. Unless islands appear simultaneously and are located the same distance from the nearest landmass, this effect will influence each island differently, even islands that share exactly the same geoenvironmental dynamics.

The non-equivalence of islands in an archipelago has consequences for some common practices used to evaluate the GDM. First, using a space-for-time substitution (where nearby islands of different ages are considered representative of different times in the life cycle of an island; Borregaard et al., 2017) may be flawed and should thus be avoided. Old and young islands of the same size may experience different archipelago effects during their lifetime and should thus not be considered as representative of different stages of the same island life cycle. Our analysis of archipelagos with different structures (Fig. 4) showed that differences in species richness trajectories between islands are especially large - and thus that the space-for-time substitution approach is probably 
not suitable - for remote archipelagos in which the islands are close to one another in comparison to the dispersal distance of the organisms considered (i.e. conditions for a strong archipelago effect), and with intermediate time intervals of island emergence (i.e. condition for a strong history effect meaning the archipelago effect will influence each island differently).

The second consequence for the practices usually used to evaluate the GDM is that area and time, the two explanatory variables usually used to characterise island biodiversity patterns in the GDM framework (Whittaker et al., 2008; Borregaard et al., 2017), may actually not be sufficient: the structure and history of the whole archipelago, i.e. the archipelago and history effects, should also be included in the analyses. The archipelago effect is accounted for indirectly in statistical analyses when evaluating the GDM with mixedeffects models where archipelago identity is considered as a random variable (Bunnefeld and Phillimore, 2012; Borregaard et al., 2017). Our results indicate that deviations from the GDM predictions may be explained by the strength of inter-island migration, the dissimilarity of the migrant pool from the local recipient community, and their variations over time. Therefore, we suggest it would be useful to incorporate fixed effect variables that reflect these biological processes affected by the spatial and temporal structure of the archipelago. Again, we expect this approach to be especially advantageous for remote archipelagos where the islands are located close to one another and emerge at intermediate time intervals.

A first step in this direction was taken by Cabral et al. (2014) and Triantis et al. (2015). Cabral et al. (2014) analysed diversity data from vascular plants across several archipelagos, and included variables characterising the spatial structure of the archipelagos in their analyses (e.g. the number of islands, mean inter-island distance). Their finding are consistent with our predictions, except for one: they found that within-archipelago structure has only a weak effect on local diversity, whereas we predict the contrary. This discrepancy may be explained by the method used by Cabral et al. (2014) to estimate local diversity (i.e. the average number of species per island in each archipelago, not the actual local diversity) which ignores the fact that within an archipelago, every island has a different set of neighbour islands, a factor we show to be extremely important to explain local diversity patterns. In other words, Cabral et al. (2014) accounted for the archipelago effect, but not for how it can combine with the history effect. Triantis et al. (2015) also analysed island biodiversity data from several archipelagos using variables characterising the spatial structure of the archipelagos. However, these authors did not include a history effect since they only considered the current state of the archipelagos. Thus, these promising approaches could be improved by accounting for variations in the archipelago effect over time (i.e. also including the history effect).

Using a different approach (comparison of observed data with data simulated with an agent-based model), Jõks et al. (2021) studied the effect of the geological history of several archipelagos and of sea level fluctuations on current biodiversity patterns. Inter-island migration is included in a similar way in their study and in our model (i.e., migration depends on the size of the original population and on the size of the destination island), but Jõks et al. (2021) did not include its evolutionary consequences where migration 
both triggers divergence and delays speciation (i.e. they modelled a restricted archipelago effect). The authors concluded that archipelago history may affect current biodiversity patterns to different degrees depending on the structure of the archipelago and on the diversity metrics considered. Despite the differences in model assumptions, our conclusions are thus in line with theirs, again underlining the advantage of taking the history of the archipelago into account to understand biodiversity patterns.

Our analysis of archipelagos with different structures led to some predictions of biodiversity patterns that appeared largely supported by empirical data. Recently, Ali and Meiri (2019) found that terrestrial reptile biodiversity on individual islands in oceanic archipelagos that are difficult to reach (for example because they are located far from the mainland) originates mainly from intra-archipelago dispersal, followed by within-island anagenetic events. This result is consistent with our prediction that the relative influence of the archipelago effect will be stronger on remote archipelagos than on those that are located close to the mainland. In their study of island bird biodiversity, Valente et al. (2020) found a positive effect of archipelago isolation on rates of anagenesis, a result that is in line with our prediction. In the case of steppingstone archipelagos, we predicted higher diversity on islands that are located centrally compared to islands located at the periphery of the archipelago. Several empirical studies have observed biodiversity patterns consistent with this prediction although they argued that this was a possible effect of the chronology of island emergence (Gillespie, 2004; Cameron et al., 2013). Theoretical investigations by Gascuel et al. (2016) offered another possible explanation: diversity is higher on centrally located islands because of their greater connectivity to other islands, which strengthens the archipelago effect they experience. Our results suggest that this biodiversity pattern could be best explained by a combination of the two previous explanations. We indeed found that (i) the diversity dynamics of geographically similarly located islands (symmetrically to the centre of the archipelago) depends on the time of their emergence (Fig. 5), as expected if the chronology of island emergence is important, and that (ii) centrally located islands host more species even when the islands appear simultaneously (Fig. S2.10), as expected if there is an important archipelago effect.

Deviations from the GDM expectations could result from non-equilibrium biodiversity dynamics (Warren et al., 2015). The debate about whether island biodiversity is at equilibrium is still not over as different studies obtained opposing results, without converging towards a single explanation for these discrepancies (Heaney, 2000; Rabosky and Glor, 2010; Bonnet-Lebrun et al., 2017; Marta et al., 2019; Valente et al., 2017, 2020). Unfortunately, our model cannot help solve this issue: the invariant observation of equilibrium dynamics in our results does not prove that non-equilibrium dynamics do not exist. Our results nevertheless shed some light on the debate: we observed equilibrium diversity dynamics which have the characteristics of non-equilibrium dynamics, i.e., species richness and the speciation rate peaked later than species carrying capacity (Fig. 2 and Fig. 3), a pattern only generated by the archipelago effect. Confusion and misinterpretation of the signatures of non-equilibrium dynamics and of the archipelago effect varying over time are therefore possible. This 
again suggests it may be essential to take details of spatio-temporal structure of the whole archipelago into account to understand insular biodiversity patterns.

\section{Data availability statement}

The code for numerical simulations is available on Zenodo under the DOI 10.5281/zenodo.4925696 (https://doi.org/10.5281/zenodo.4925696).

\section{Acknowledgements}

The code for numerical simulations is available on Zenodo under the DOI 10.5281/zenodo.4925696 (https://doi.org/10.5281/zenodo.4925696).We thank Kenneth F. Rijsdijk for helpful discussions about the configuration of archipelagos. Some of the analyses were run on the EDB-Calc cluster (which uses software developed by the Rocks Cluster Group at the San Diego Supercomputer Center, University of California) at the Évolution $\&$ Diversité Biologique laboratory. We thank Pierre Solbès for support with the EDB-Calc system. We thank the Institut National de Recherche pour l'Agriculture, l'alimentation et l'Environnement (INRAE) MIGALE bioinformatics facility (MIGALE, INRAE, 2020. Migale bioinformatics Facility, doi: 10.15454/1.5572390655343293E12) for providing computing resources. Financial support was provided by the French Laboratory of Excellence project 'TULIP' (ANR-10-LABX-41), and by the Fondation pour la Recherche sur la Biodiversité through its Centre for Synthesis and Analysis of Biodiversity (ISLANDS project). JC was supported by the INCEPTION programme (ANR-16-CONV-0005), College de France and the Bettencourt École Doctorale FIRE-CRI-Programme. Permits were not required for this work.

\section{References}

Ali, J. R. and Meiri, S. (2019). Biodiversity growth on the volcanic ocean islands and the roles of in situ cladogenesis and immigration: case with the reptiles. Ecography, 42:989-999.

Bonnet-Lebrun, A.-S., Manica, A., Eriksson, A., and Rodrigues, A. S. L. (2017). Empirical phylogenies and species abundance distributions are consistent with preequilibrium dynamics of neutral community models with gene flow. Evolution, 71(5):1149-1163.

Borregaard, M. K., Amorim, I. R., Borges, P. A. V., Cabral, J. S., FernándezPalacios, J. M., Field, R. R., Heaney, L. R., Kreft, H., Matthews, T. J., Olesen, J. M., Price, J., Rigal, F., Steinbauer, M. J., Triantis, K. A., Valente, L., Weigelt, P., and Whittaker, R. J. (2017). Oceanic island biogeography through the lens of the general dynamic model: assessment and prospect. Biol Rev Camb Philos Soc, 92(2):830-853. 
Borregaard, M. K., Matthews, T. J., and Whittaker, R. J. (2016). The general dynamic model: towards a unified theory of island biogeography? Glob Ecol Biogeogr, 25(7):805-816.

Brown, J. H. and Kodric-Brown, A. (1977). Turnover rates in insular biogeography: effect of immigration on extinction. Ecology, 58(2):445-449.

Bunnefeld, N. and Phillimore, A. B. (2012). Island, archipelago and taxon effects: mixed models as a means of dealing with the imperfect design of nature's experiments. Ecography, 35:15-22.

Cabral, J. S., Weigelt, P., Kissling, W. D., and Kreft, H. (2014). Biogeographic, climatic and spatial drivers differentially affect alpha-, beta- and gammadiversities on oceanic archipelagos. P Roy Soc Lond B Bio, 281:20133246.

Cabral, J. S., Whittaker, R. J., Wiegand, K., and Kreft, H. (2019). Assessing predicted isolation effects from the general dynamic model of island biogeography with an eco-evolutionary model for plants. J Biogeogr, 46:1569-1581.

Cameron, R. A. D., Triantis, K. A., Parent, C. E., Guilhaumon, F., Alonso, M. R., Ibanez, M., de Frias Martins, A. M., Ladle, R. J., and Whittaker, R. J. (2013). Snails on oceanic islands: testing the general dynamic model of oceanic island biogeography using linear mixed effect models. $J$ Biogeogr, 40:117-130.

Casquet, J., Bourgeois, Y. X. C., Cruaud, C., Gavory, F., Gillespie, R. G., and Thébaud, C. (2015). Community assembly on remote islands: a comparison of Hawaiian and Mascarene spiders. J Biogeogr, 42(1):39-50.

Chen, X., Jiao, J., and Tong, X. (2011). A generalized model of island biogeography. Sci China Life Sci, 54(11):1055-1061.

Clark, J. S., Silman, M., Kern, R., Macklin, E., and HilleRisLambers, J. (1999). Seed dispersal near and far: Patterns across temperate and tropical forest. Ecology, 80:1475-1494.

Gascuel, F., Laroche, F., Bonnet-Lebrun, A.-S., and Rodrigues, A. S. (2016). The effects of archipelago spatial structure on island diversity and endemism: predictions from a spatially-structured neutral model. Evolution, 70(11):2657-2666.

Gillespie, D. T. (1977). Exact stochastic simulation of coupled chemical reactions. J Phys Chem, 81:2340-2361.

Gillespie, R. (2004). Community assembly through adaptive radiation in Hawaiian spiders. Science, 303:356-359.

Gilpin, M. E. and Diamond, J. M. (1976). Calculation of immigration and extinction curves from the species-area-distance relation. Proc Natl Acad Sci USA, 73(11):4130-4134. 
Haydon, D., Radtkey, R. R., and Pianka, E. R. (1993). Experimental biogeography: interactions between stochastic, historical, and ecological processes in a model archipelago. In Ricklefs, R. E. and Schluter, D., editors, Species Diversity in Ecological Communities: Historical and Geographical Perspectives, pages 117-130. University of Chicago Press, Chicago.

Heaney, L. R. (2000). Dynamic disequilibrium: a long-term, large-scale perspective on the equilibrium model of island biogeography. Glob Ecol Biogeogr, 9:59-74.

Heaney, L. R., Balete, D. S., and Rickart, E. A. (2013). Models of oceanic island biogeography: changing perspectives on biodiversity dynamics in archipelagoes. Front Biogeogr, 5:249-257.

Hubbell, S. P. (2001). The Unified Neutral Theory of Biodiversity and Biogeography. Princeton University Press, Princeton, NJ, USA.

Jõks, M., Kreft, H., Weigelt, P., and Pärtel, M. (2021). Legacy of archipelago history in modern island biodiversity - An agent-based simulation model. Global Ecol Biogeogr, 30(1):247-261.

Jõks, M. and Pärtel, M. (2018). Plant diversity in oceanic archipelagos: realistic patterns emulated by an agent-based computer simulation. Ecography, 42:1-15.

Kisel, Y. and Barraclough, T. G. (2010). Speciation has a spatial scale that depends on levels of gene flow. Am Nat, 175:316-334.

Lomolino, M. V. (2000). A call for a new paradigm of island biogeography. Glob Ecol Biogeogr, 9:1-6.

Losos, J. B. and Ricklefs, R. E. (2009). Adaptation and diversification on islands. Nature, 457:830-836.

MacArthur, R. H. and Wilson, E. O. (1963). An equilibrium theory of insular zoogeography. Evolution, 17:373-387.

MacArthur, R. H. and Wilson, E. O. (1967). The Theory of Island Biogeography. Princeton University Press, Princeton, NJ.

Marta, S., Lacasella, F., Cesaroni, D., and Sbordoni, V. (2019). Effects of Holocene climate changes on alpine ecosystems: Nonequilibrium dynamics drive insect species richness on alpine islands. J Biogeogr, 46:2248-2259.

R Core Team (2017). R: A Language and Environment for Statistical Computing. R Foundation for Statistical Computing, Vienna, Austria.

Rabosky, D. L. and Glor, R. E. (2010). Equilibrium speciation dynamics in a model adaptive radiation of island lizards. Proc Natl Acad Sci USA, 107:22179-22183.

Ricklefs, R. E. (2004). A comprehensive framework for global patterns in biodiversity. Ecol Lett, 7:1-15. 
Rosindell, J. and Phillimore, A. B. (2011). A unified model of island biogeography sheds light on the zone of radiation. Ecol Lett, 14:552-560.

Shaw, K. L. and Gillespie, R. G. (2016). Comparative phylogeography of oceanic archipelagos: hotspots for inferences of evolutionary process. Proc Natl Acad Sci USA, 113(29):7986-7993.

Steinbauer, M., Dolos, K., Field, R., Reineking, B., and Beierkuhnlein, C. (2013). Re-evaluating the general dynamic theory of oceanic island biogeography. Front Biogeogr, 5:185-194.

Triantis, K. A., Economo, E. P., Guilhaumon, F., and Ricklefs, R. E. (2015). Diversity regulation at macro-scales: species richness on oceanic archipelagos. Glob Ecol Biogeogr, 24:594-605.

Triantis, K. A., Whittaker, R. J., Fernández-Palacios, J. M., and Geist, D. J. (2016). Oceanic archipelagos: a perspective on the geodynamics and biogeography of the world's smallest biotic provinces. Front Biogeogr, $8(2): \mathrm{e} 29605$.

Valente, L., Etienne, R., and Phillimore, A. (2014). The effects of island ontogeny on species diversity and phylogeny. $P$ Roy Soc Lond B Bio, 281:20133227.

Valente, L., Illera, J. C., Havenstein, K., Pallien, T., Etienne, R. S., and Tiedemann, R. (2017). Equilibrium bird species diversity in Atlantic islands. Curr Biol, 27(11):1660-1666.

Valente, L., Phillimore, A. B., Melo, M., Warren, B. H., Clegg, S. M., Havenstein, K., Tiedemann, R., Illera, J. C., Thébaud, C., Aschenbach, T., and Etienne, R. S. (2020). A simple dynamic model explains the diversity of island birds worldwide. Nature, 579:92-96.

Warren, B. H., Simberloff, D., Ricklefs, R. E., Aguilée, R., Condamine, F. L., Gravel, D., Morlon, H., Mouquet, N., Rosindell, J., Casquet, J., Conti, E., Cornuault, J., Fernández-Palacios, J. M., Hengl, T., Norder, S. J., Rijsdijk, K. F., Sanmartín, I., Strasberg, D., Triantis, K. A., Valente, L. M., Whittaker, R. J., Gillespie, R. G., Emerson, B. C., and Thébaud, C. (2015). Islands as model systems in ecology and evolution: prospects fifty years after MacArthur-Wilson. Ecol Lett, 18:200-217.

Warren, B. H., Strasberg, D., Bruggemann, J. H., Prys-Jones, R. P., and Thébaud, C. (2010). Why does the biota of Madagascar region have such a strong Asiatic flavor? Cladistics, 26:526-538.

Waters, J. M., Emerson, B. C., Arribas, P., and McCulloch, G. A. (2020). Dispersal reduction: causes, genomic mechanisms, and evolutionary consequences. Trends Ecol Evol, 35(6):512-522.

Whittaker, R. J. (2000). Scale, succession and complexity in island biogeography: are we asking the right questions? Glob Ecol Biogeogr, 9:75-85. 
Whittaker, R. J. and Fernández-Palacios, J. M. (2007). Island Biogeography: Ecology, Evolution and Conservation (2nd edn). Oxford University Press, Oxford, UK.

Whittaker, R. J., Fernández-Palacios, J. M., Matthews, T. J., Borregaard, M. K., and Triantis, K. A. (2017). Island biogeography: taking the long view of nature's laboratories. Science, 357(6354):eaam8326.

Whittaker, R. J., Fernández-Palacios, J. M., Matthews, T. J., Rigal, F., and Triantis, K. A. (2018). Archipelagos and meta-archipelagos. Front Biogeogr, 10:e41470.

Whittaker, R. J., Triantis, K. A., and Ladle, R. J. (2008). A general dynamic theory of oceanic island biogeography. J Biogeogr, 35:977-994.

\section{Author contributions}

RA designed the study, performed the research and wrote the manuscript. FP, MS and JC analysed preliminary versions of the model. CT discussed the results. All the authors contributed to the final version of the manuscript.

\section{Handling Editor}

Luis Valente.

\section{Biosketch}

RA studies ecological and evolutionary processes involved in adaptation and diversification. He focuses on spatially structured populations, and on how changes in spatial structures affect eco-evolutionary processes. He aims to model these processes at large time scales to better understand biodiversity patterns and dynamics. 


\title{
Supporting Information for
}

\author{
Biogeographic drivers of community assembly \\ on oceanic islands: the importance of \\ archipelago structure and history
}

Robin Aguilée, Félix Pellerin, Maxence Soubeyrand,
Jeremy Choin and Christophe Thébaud

Published in Journal of Biogeography, 2021, 48(10): 2616-2628 doi: $10.1111 /$ jbi.14228

\section{Appendix S1 Detailed methods}

\section{$1.1 \quad$ Landscape dynamics}

Following Valente et al. (2014), the island carrying capacity function on island $i$ is defined as

$$
K_{i}(t)=\frac{K_{\max }\left(\frac{t-t_{\text {start }}}{t_{\text {life }}}\right)^{g}\left(1-\frac{t-t_{\text {start }}}{t_{\text {life }}}\right)^{h}}{\left(\frac{g}{g+h}\right)^{g}\left(\frac{h}{g+h}\right)^{h}}
$$

where $g=m \frac{\left(t_{\max }-t_{\text {start }}\right)}{(1+m)\left(t_{\text {life }} / 10\right)}, h=g / m, m=\frac{p_{\max }}{1-p_{\max }}, K_{\max }$ is the maximum of the carrying capacity function, $t_{\text {start }}$ is the birth date of the focal island, $t_{\text {life }}$ is its lifetime, $p_{\max }$ is the proportion of the island lifetime at which the maximum carrying capacity is reached, and $t_{\max }=t_{\text {start }}+p_{\max } t_{\text {life }}$ is the corresponding date. The values of the parameters can differ between islands in the archipelago.

\subsection{Population dynamics}

The model is ecologically neutral within the archipelago, which means that the species identity of an individual in the archipelago has no effect on the rate at which events affect the individual. Individual rates may however depend on the environment. Consequently, the rates of events are identical for all individuals on a given island, but may vary between islands.

On an island $i$ with carrying capacity $K_{i}$ and population size $N_{i}$, each individual dies at rate $\delta(i)=\beta \exp \left(-\gamma\left(1-N_{i} / K_{i}\right)\right)$. The parameter $\gamma$ adjusts the strength of density-dependence: the smaller the $\gamma$, the more likely the population may not have reached its carrying capacity. Because $K_{i}$ and $N_{i}$ vary over time, the death rate $\delta$ may also change over time.

When an individual starts a differentiating lineage via anagenesis or cladogenesis, it becomes a "variant" of the same species. Several variants of the 
same species may exist on different islands (due to anagenesis and/or cladogenesis) and on the same island (due to cladogenesis). Following Gascuel et al. (2016), when a variant appears we assign it a "speciation clock" initialised at $\tau$, the minimum time required for speciation, and the speciation clock decreases as time goes by. When a migrant arrives in an island where the species is already present, we randomly choose which local individual of the same species the migrant hybridises with, independently of the variant identity. The migrant becomes an individual of the population with which it has hybridised (i.e. we change its variant identity if it was a different variant of the same species), and we delay the speciation clock of the population with whom the migrant has hybridised by $\alpha / N_{\mathrm{v}}$ where $N_{\mathrm{v}}$ is the size of this population. One hybridisation event therefore delays less speciation in large populations than in small populations, which models the stronger dilution of migrant genes in larger populations. The value of $\alpha$ makes it possible to adjust the strength with which dispersal delays speciation. Its default value is 100 , which means that one migrant in a population of 100 individuals delays speciation by one generation. A larger value of $\alpha$ corresponds to a longer delay. The state of the speciation clock is inherited by the offspring from the parent at birth events. When the speciation clock of a variant reaches zero (note that variants may go extinct before their speciation clock reaches zero) all individuals of the variant become a new species, never seen before on the mainland or in the archipelago. Speciation is irreversible, gene flow has no effect once speciation is achieved.

We include the target area effect (Gilpin and Diamond, 1976) in the modelling of dispersal. Following MacArthur and Wilson (1967), at each dispersal event we evaluate the probability of going in the right direction (i.e. allowing migrants to arrive on the island after a linear journey) as the ratio of the range of departure directions allowing to reach the destination to all possible directions. We assume that the destination is a circular island, located far enough from the source so that its curvature can be ignored, as illustrated in Fig. S1.1. The probability of dispersing in the right direction is therefore $p(K, d)=\frac{2 r}{2 \pi d}=\frac{\sqrt{K}}{\pi^{3 / 2} d}$ where $K$ is the size of the destination estimated by its carrying capacity and $d$ is the distance between the centre of landmasses. We assume that there is no masking effect of one island behind any other island.

We use the power-law dispersal kernel from Clark et al. (1999): the probability of successful dispersal between two landmasses separated by a distance $d$ is $f(c, d)=\frac{c}{2 \pi\left(c^{2}+d^{2}\right)^{3 / 2}}$ where $c$ is the parameter controlling the dispersal ability of the species.

Taking into account that each individual emigrates at rate $\mu$, the dispersal rate from landmass $i$ (either the mainland or an island) to island $j$ (no return to the source, i.e. $j \neq i)$ is $\lambda(i, j)=\mu N_{i} p\left(K_{j}, d\right) f(c, d)$ where $N_{i}$ is the population size of the source and $K_{j}$ is the carrying capacity of the destination.

\section{$1.3 \quad$ Null models}

The null model "island archipelago-equivalent" is a single isolated island whose carrying capacity at all times equals the sum of the carrying capacities of all the islands in the corresponding archipelago. To compute the probability $p$ of going in the right direction to reach an island when dispersing (see Section 1.2 


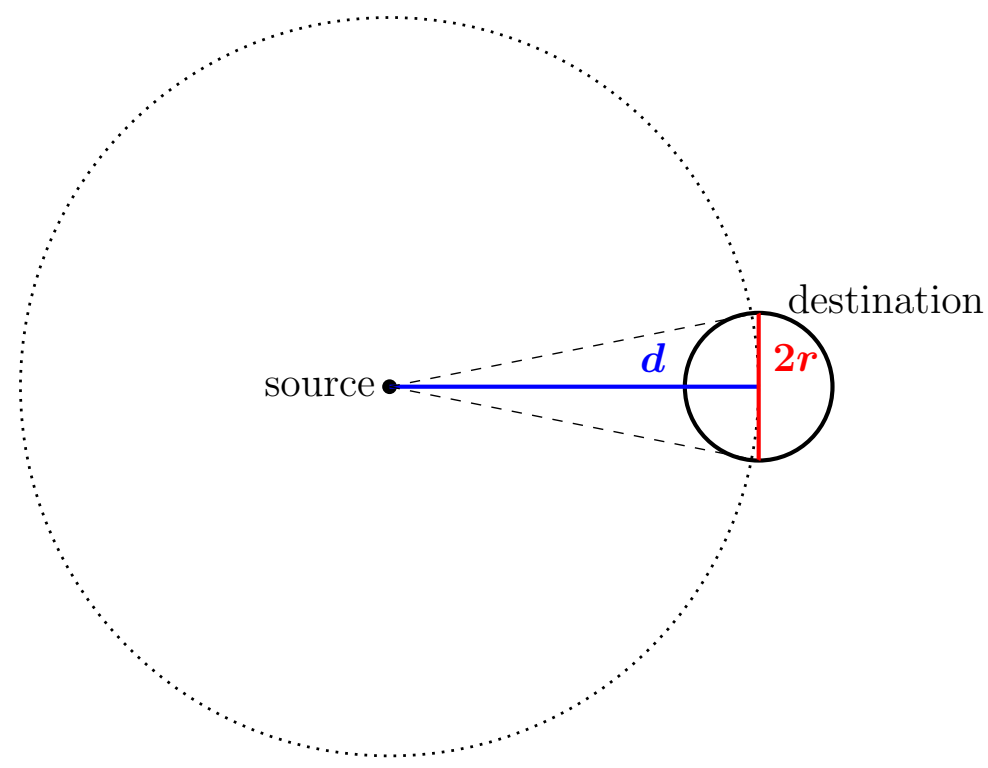

Figure S1.1: Assumptions used to compute of the probability to disperse in the right direction to reach a given island.

in this appendix), we do not assume that the island archipelago-equivalent is a circular island as we would normally, because this would lead to a lower probability $p$ than for the corresponding archipelago. Instead, we assume that $p$ in the island archipelago-equivalent model equals $p$ in the corresponding archipelago. Therefore, comparing the results of the baseline model at the scale of the archipelago with the island archipelago-equivalent model allows us to extract the consequences of intra-archipelago processes only.

In the null "static" models, for each archipelago configuration sampled, we run the sub-model routine with a static landscape for $5.10^{4}$ generations. We observed in each replicate simulation (checked by hand) that species richness reached its equilibrium value within the first 2.5.10 ${ }^{4}$ generations. Then we compute the equilibrium value for species richness as its average over the next 2.5.10 ${ }^{4}$ generations (i.e. from generation $2.5 .10^{4}$ to generation $5.10^{4}$ ).

\subsection{Numerical simulation methods}

We simulate the model forward in time using Gillespie (1977)'s stochastic algorithm in continuous time. At any time, four events can happen in the archipelago: the birth of an individual at rate $N \beta$ (where $N$ is the size of the total population of the archipelago), the death of an individual at rate $\sum_{i}\left(N_{i} \delta(i)\right)$ (where $N_{i}$ is the size of the population on island $i$ ), the initiation of a cladogenesis event at rate $N \sigma$, and the dispersal of an individual at rate $\sum_{i} \sum_{j \neq i} \lambda(i, j)$ ( $i$ being the mainland or an island, and $j$ being an island). The rate at which each event occurs is detailed in Section 1.2 in this appendix. We pick the time until the next event from an exponential distribution with mean $1 /\left(N \beta+\sum_{i}\left(N_{i} \delta(i)\right)+N \sigma+\sum_{i} \sum_{j \neq i} \lambda(i, j)\right)$. The occurring event is then randomly chosen proportionally to the rate of each possible event. Completion of speciation is not an event in the sense of Gillespie (1977)'s algorithm because it does not occur stochastically with a defined rate. 
Because the carrying capacity of the islands changes continuously, the landscape changes at each event. However, we update the landscape only once every generation. If a speciation clock reaches zero, we make the speciation event happen at the time the landscape is updated. This means that, in our simulation algorithm, the landscape changes and the completion of speciation events can be delayed by up to one generation compared to the exact algorithm. This approximation allows us to save considerable computing time and has no effect on the results (Fig. S1.2).

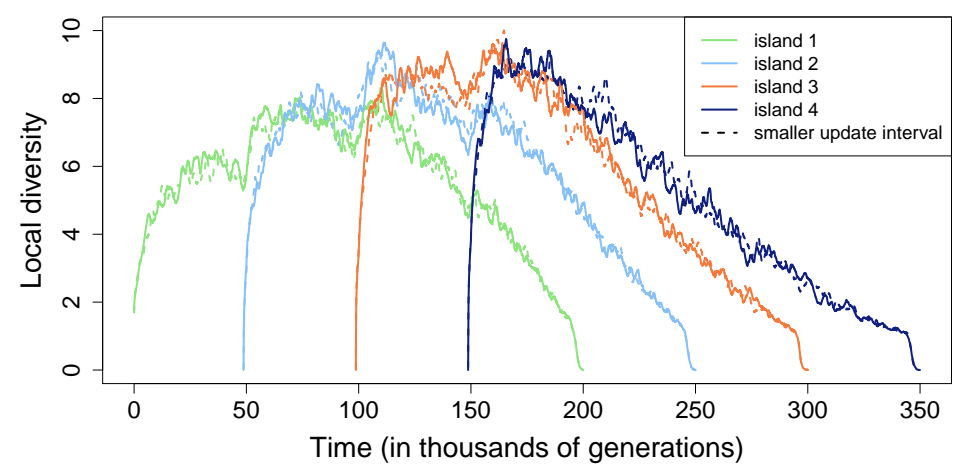

Figure S1.2: Mean dynamics over 25 replicate simulations of local diversity in the baseline model (solid lines) and in the same model but in which the landscape is updated every 0.1 generation (dashed lines) instead of every generation. The two cases are superimposed. Default parameter values (Table S2.1).

We measure time in generations: the generation time is equal to one time unit of the simulation real time divided by the per-capita death rate. As the death rate can vary over time, we approximate it by the constant per-capita birth rate, which is expected to have the same value when the population is at its ecological equilibrium. This assumption is reasonable since our results show that simulated communities are indeed at equilibrium (Fig. 2b,c).

The core of the simulation algorithm is coded with the $\mathrm{C}$ language. We use the GNU Scientific Library (Galassi et al., 2009) for random number generation. We generate the mainland composition with a re-coding in $\mathrm{C}$ of the rand.neutral() function from the $\mathrm{R}$ package 'untb' (Hankin, 2009). The $\mathrm{C}$ code is embedded in a script written in the $\mathrm{R}$ language ( $\mathrm{R}$ Core Team, 2017) to allow easy replication and extension of the simulations. We analyse the outputs of the model using $\mathrm{R}$ scripts.

\subsection{Model analysis methods}

Some of the outputs characterising the state and evolution of the population are rates (dispersal rates, etc.). These outputs are measures of the real rates (i.e. number of observed events per time unit), not measures of the expected rates (i.e. the ones used to simulate the model).

We collect the value of the outputs every 25 generations. During this time interval, for most outputs, either one or zero event occurs (e.g. one or zero individual migrates from the mainland). This makes it difficult to visualise variations in the outputs over evolutionary significant times at a 25 - 
generation resolution and therefore difficult to interpret. Consequently, for each output, we compute its temporal mean on a sliding temporal window of size $x$ generations: at time $t$, the value of a given output corresponds to its mean from $t$ to $t+x$ (or from $t$ to the maximum time of simulation for $t+x$ greater than this maximum time). This smooths the variations of the output concerned, making it possible to visualise and interpret its variations over time. The size of the smoothing window depends on the output: an output corresponding to less frequent events requires an average over a larger window to allow visualisation of its variations. We use a smoothing window of 50 data points (i.e. average over $x=50 \times 25=1250$ generations) for all outputs, except for the speciation rates and the speciation duration for which the smoothing window is of 150 data points (3,750 generations), and except on Fig. 4c and Fig. 5 where we use a smoothing window of 200 data points (5,000 generations) to facilitate visualisation of the results. 


\section{Appendix S2 Supplementary tables and figures}

Table S2.1: Parameters and default numerical values for the baseline model.

\begin{tabular}{lll}
\hline Parameter & Definition & Default value \\
\hline$n$ & Total number of islands & 4 \\
$t_{\text {inter }}$ & Time interval between island emergence & $5.10^{4}$ \\
$t_{\text {life }}$ & Lifetime of an island & $2.10^{5}$ \\
$p_{\max }$ & Proportion of the island lifetime at which the max- & 0.2 \\
& imum carrying capacity is reached & \\
$K_{\max }$ & Maximum carrying capacity of an island & $2.10^{4}$ \\
$d_{\mathrm{c}}$ & Distance to the mainland & 150 \\
$d_{\mathrm{i}}$ & Distance between islands & 20 \\
$J$ & Metacommunity size on the mainland & $10^{6}$ \\
$\theta$ & Fundamental biodiversity number on the mainland & 20 \\
$\beta$ & Per-capita birth rate & 1 \\
$\gamma$ & Strength of mortality density-dependence & 0.1 \\
$\sigma$ & Per-capita cladogenesis initiation rate & $5.10^{-5}$ \\
$\tau$ & Minimum duration for speciation & 500 \\
$\alpha$ & Strength with which dispersal delays speciation & 100 \\
$\mu$ & Per-capita rate of emigration & 1 (from the mainland) \\
& & or 0.1 (from an island) \\
$c$ & Individual dispersal ability & 25 (from the mainland) \\
& & or 10 (from an island) \\
\hline
\end{tabular}



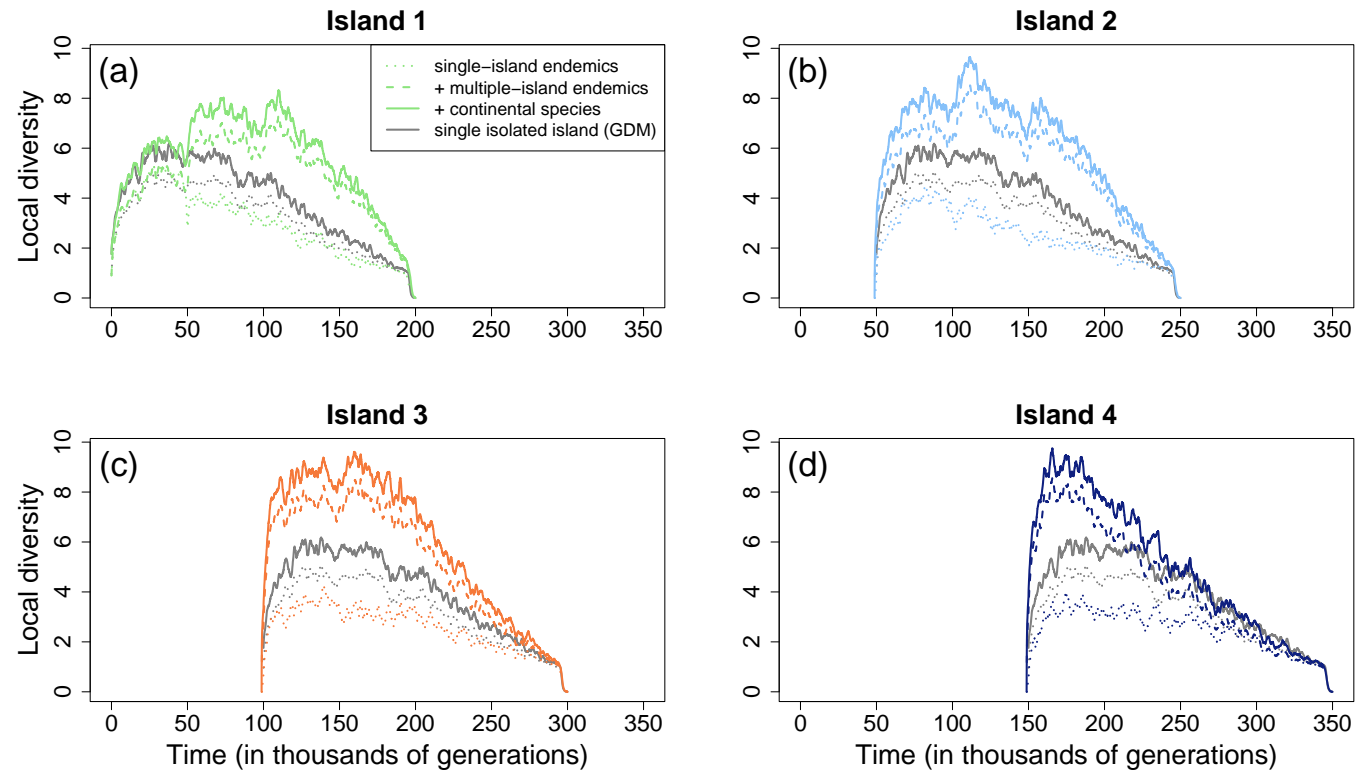

Figure S2.3: Mean dynamics over 25 replicate simulations of single-island endemic species, of all endemic species, and of all species on islands 1 to 4 (panels (a)-(d)) in the baseline model. In each panel, we also plot the dynamics of endemic species and of all species in the single isolated island model (gray curves) transposed over time so that the emergence time of the single isolated island matches the one of each island in the archipelago. Default parameter values (Table S2.1). Note that, in our model, newly formed species are necessarily initially single-island endemic species, and if they do not become extinct, they necessarily disperse to other islands sooner or later, becoming multiple-island endemic species at that time. Consequently, the number of single-island endemic species observed on each island of the archipelago may be lower than on a single isolated island, as shown here, despite the formation of more single-island endemic species on each island of the archipelago than on a single isolated island. 

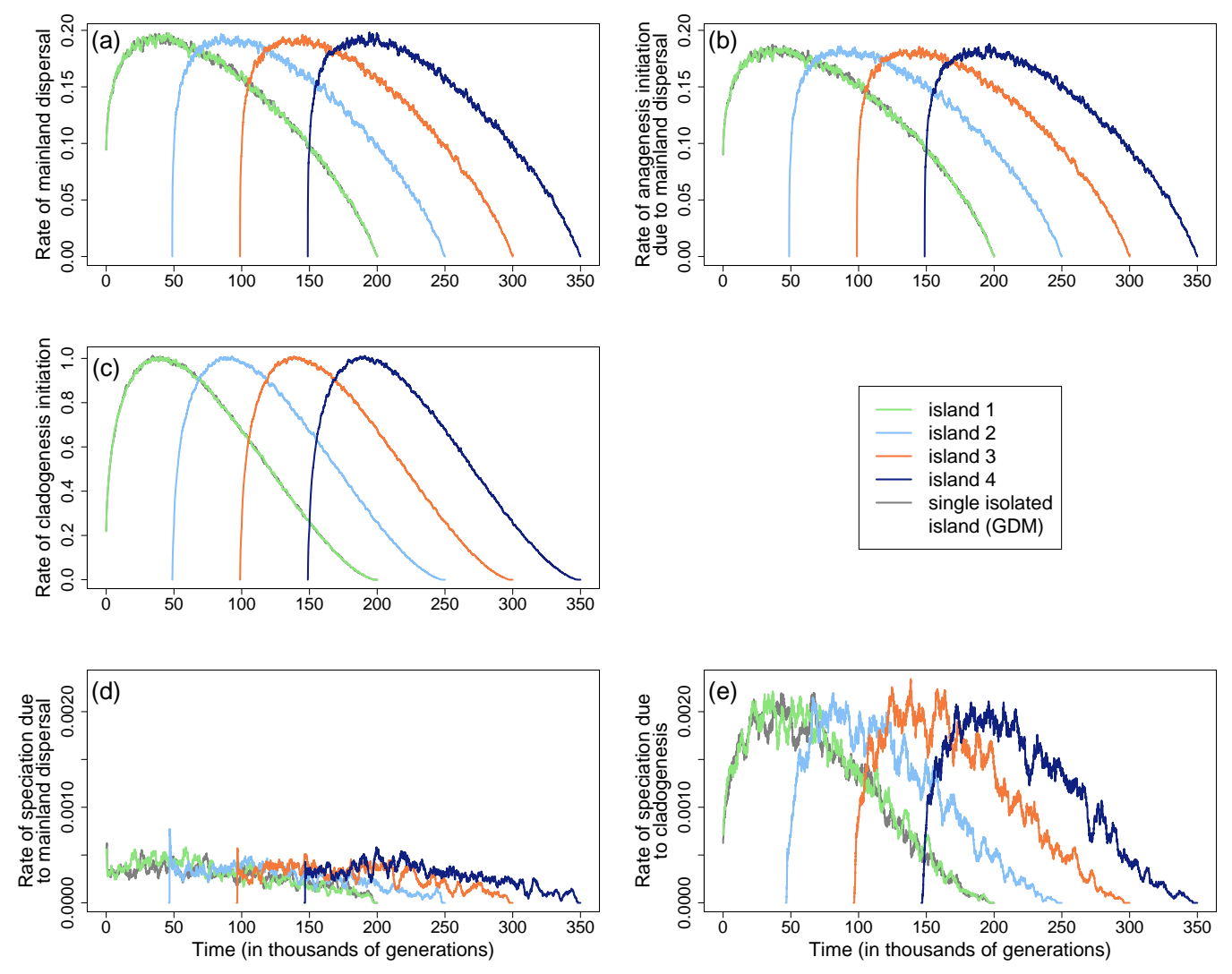

Figure S2.4: Mean dynamics over 25 replicate simulations of various outputs characterising the state and evolution of the population (see Section 2.6 in the main text) in the baseline model and in the single isolated island model. In all panels, the curve of the single isolated island is almost entirely hidden by the curve of island 1. Default parameter values (Table S2.1). Several islands coexist between times 50 and 300 (in thousands of generations; Fig. 2a). 

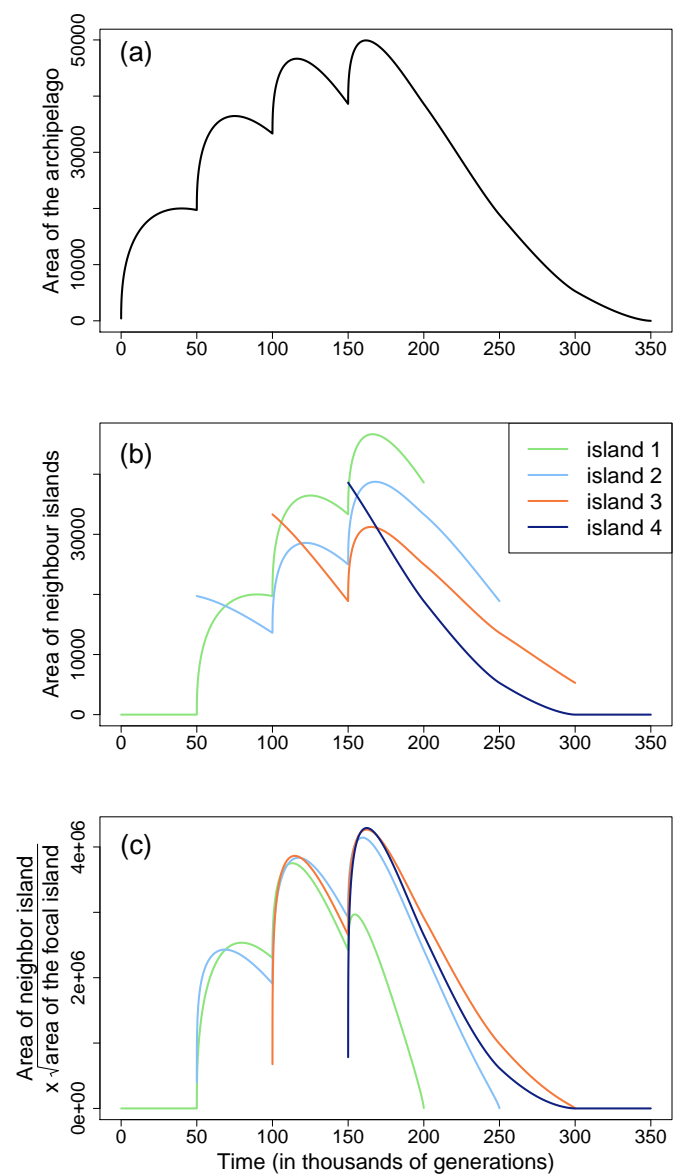

Figure S2.5: Dynamics of the total area of the archipelago (a), of cumulative size of the neighbour islands (b) and of cumulative size of the neighbour islands times the square root of the size of the focal island (c; given the model design detailed in Appendix S1, the amount of inter-island migrants received by a focal island is expected to be proportional to the cumulative size of the populations of the neighbour islands and proportional to the square root of the size of the focal island) for the baseline model. Default parameter values (Table S2.1). 

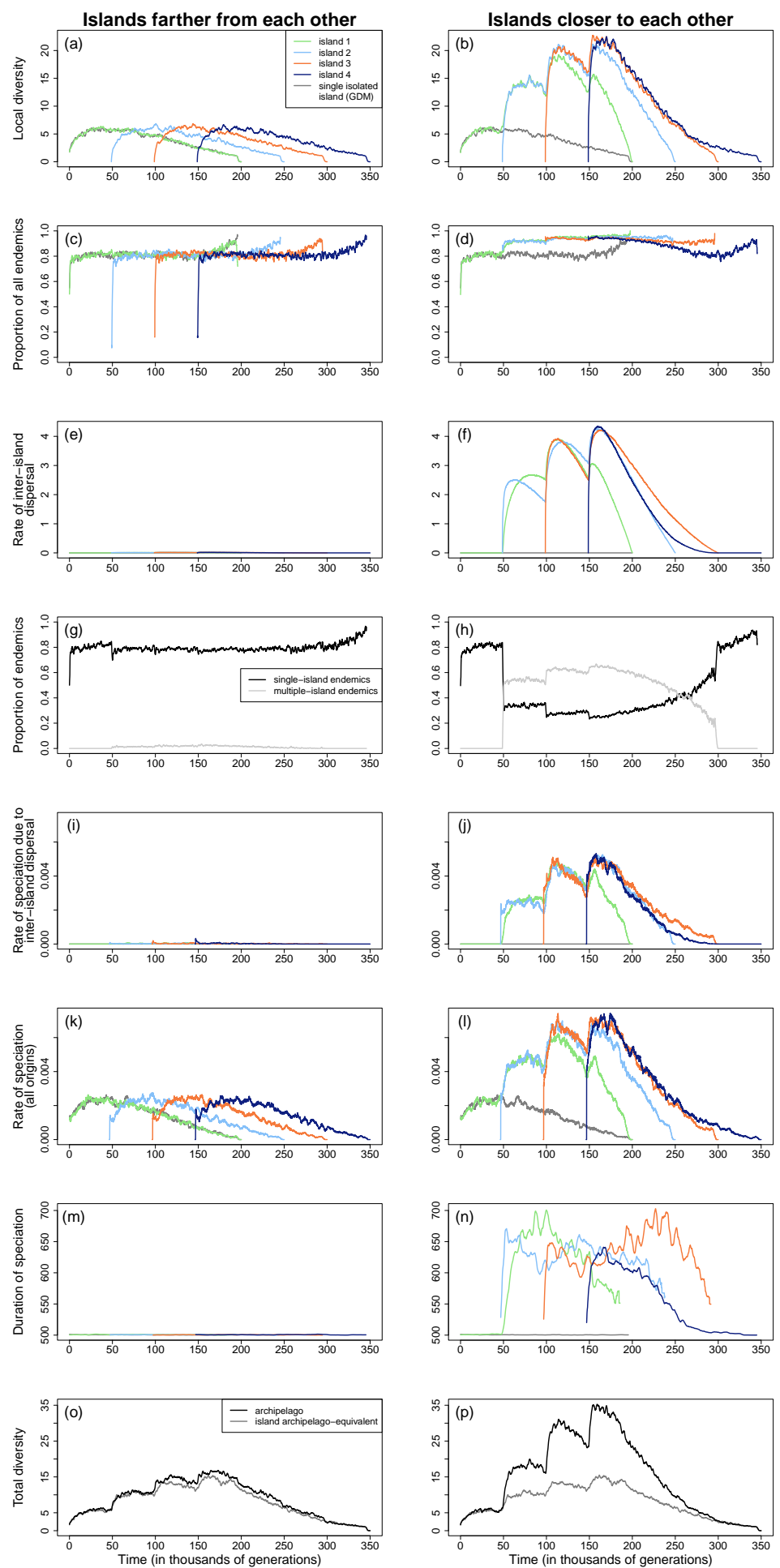

Figure S2.6: Mean dynamics over 25 replicate simulations of various outputs (see Section 2.6 in the main text) in an archipelago where the islands are located farther from one another than in the baseline model (left panels; $d_{\mathrm{i}}=$ 50 ) or closer (right panels; $d_{\mathrm{i}}=10$ ). The other parameters are set to default values (Table S2.1). Except for the fourth and last rows, the outputs are plotted for the archipelago model and for the single isolated island model. The fourth row shows the proportion of single- and multiple-island endemics among all species at the archipelago scale. The last row shows total diversity in the archipelago model and in the island archipelago-equivalent model. 

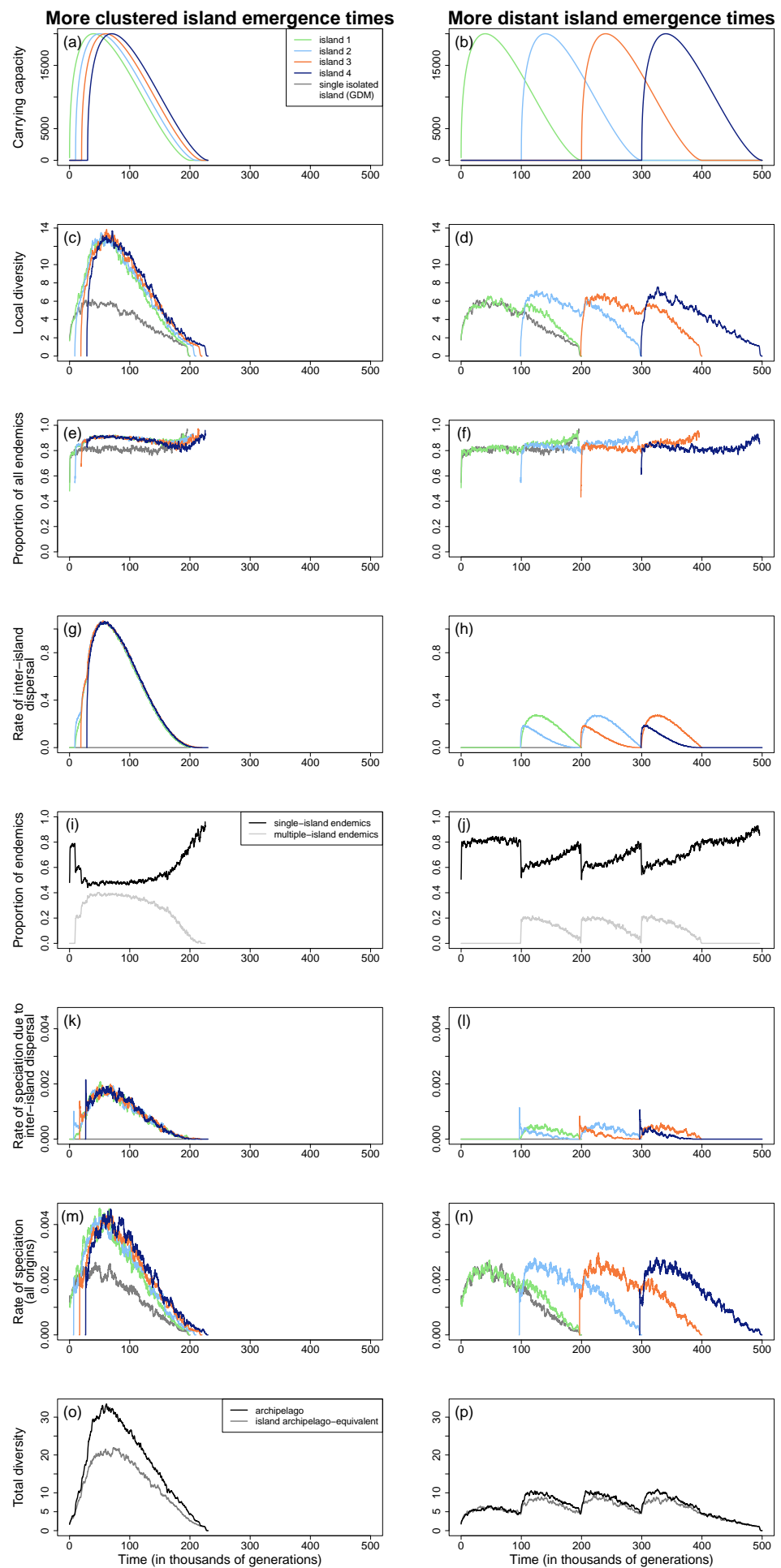

Figure S2.7: Mean dynamics over 25 replicate simulations of various outputs (see Section 2.6 in the main text) in an archipelago where islands emerge faster one after the other than in the baseline model (left panels; $t_{\text {inter }}=10^{4}$ ) or slower (right panels; $t_{\text {inter }}=10^{5}$ ). The other parameters are set to default values (Table S2.1). Except for the fifth and last rows, the outputs are plotted for the archipelago model and for the single isolated island model. The fifth row shows the proportion of single- and multiple-island endemics among all species at the archipelago scale. The last row shows total diversity in the archipelago model and in the island archipelago-equivalent model. 

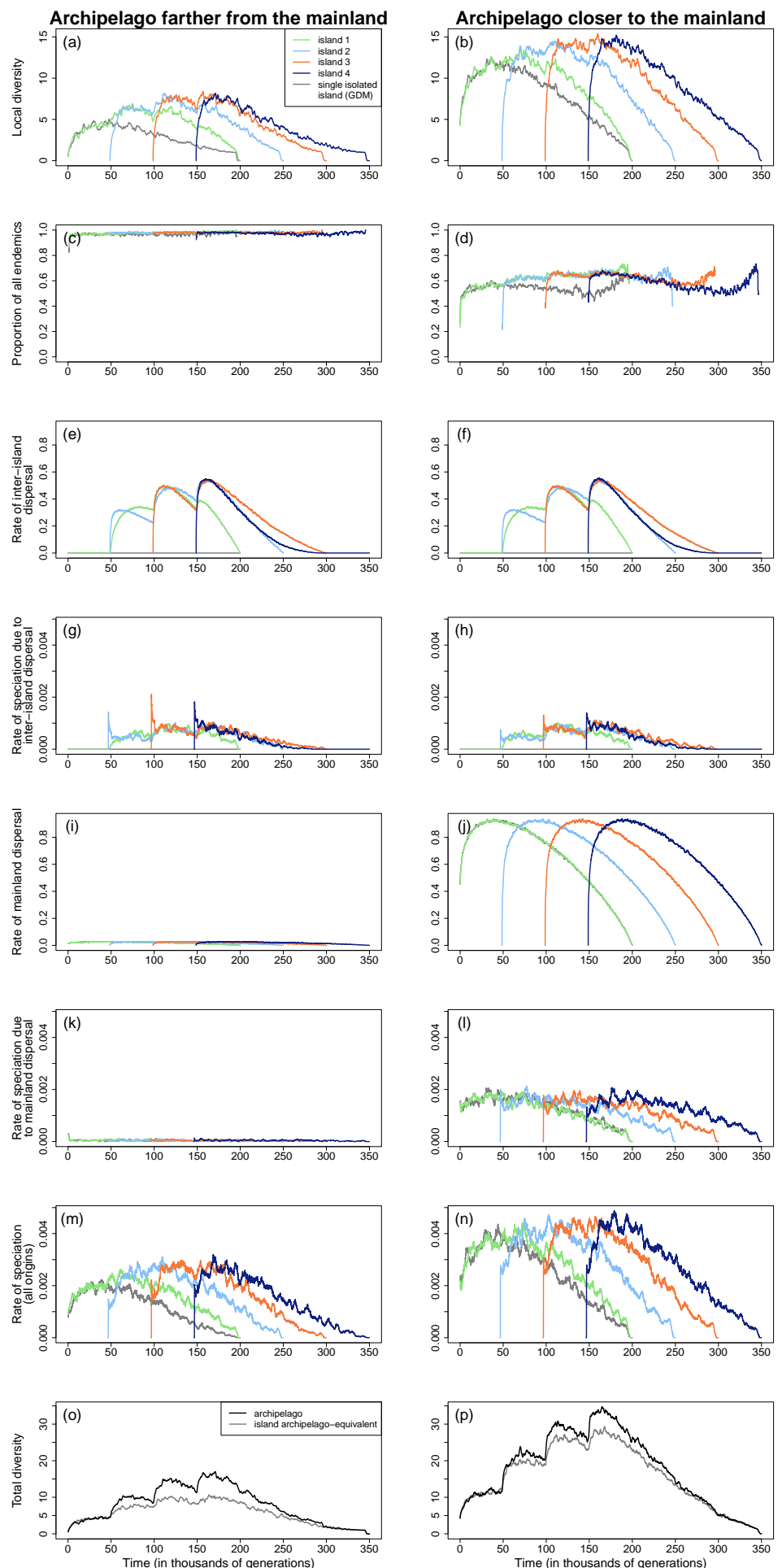

Figure S2.8: Mean dynamics over 25 replicate simulations of various outputs (see Section 2.6 in the main text) in an archipelago located farther from the mainland than in the baseline model (left panels; $d_{\mathrm{c}}=250$ ) or closer (right panels; $d_{\mathrm{c}}=100$ ). The other parameters are set to default values (Table S2.1). Except for the last row, the outputs are plotted for the archipelago model and for the single isolated island model. The last row shows total diversity in the archipelago model and in the island archipelago-equivalent model. Distance to the mainland is set for the null models like in the corresponding archipelago model. 

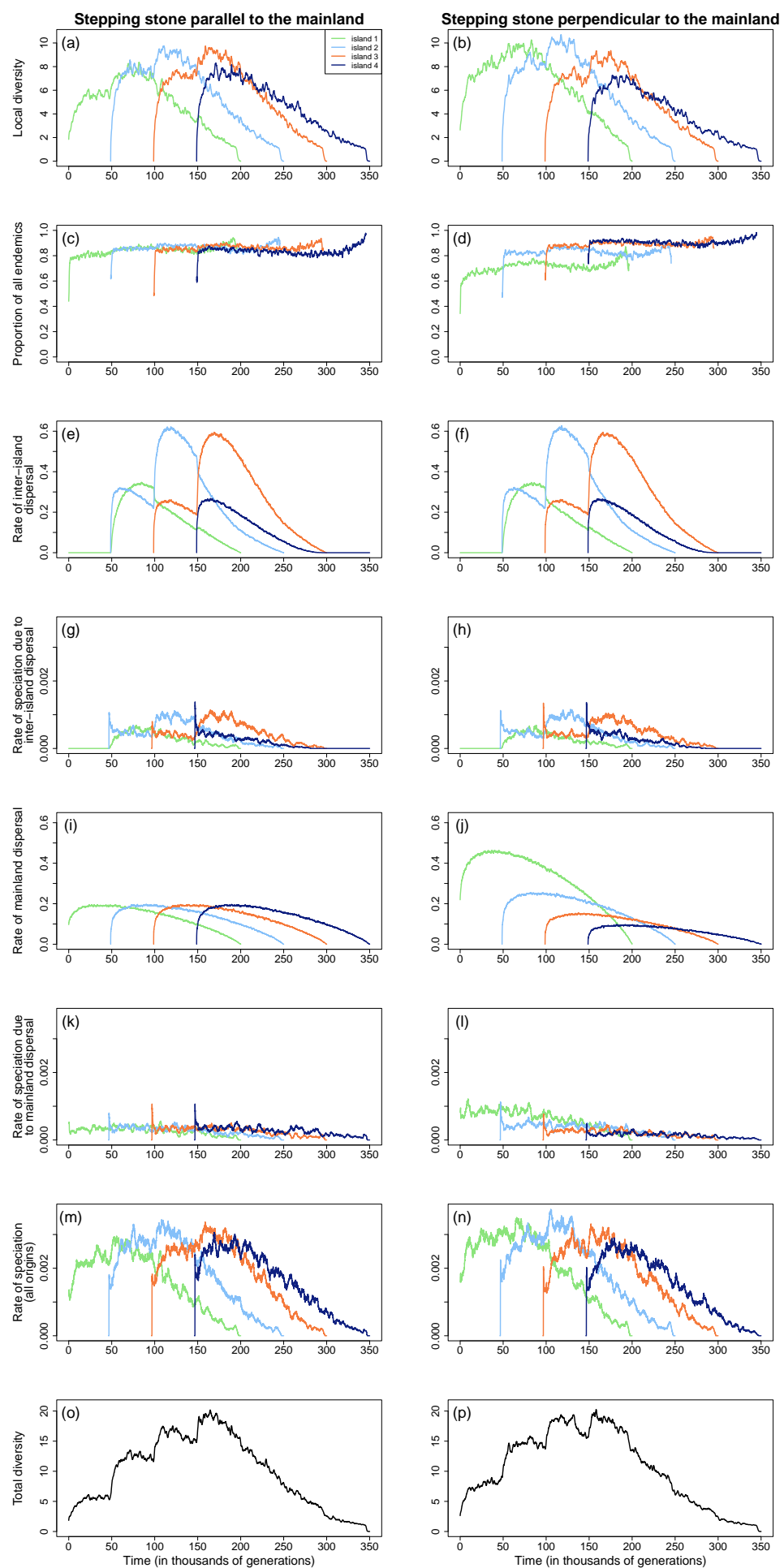

Figure S2.9: Mean dynamics over 25 replicate simulations of various outputs (see Section 2.6 in the main text) in an archipelago shaped as a linear stepping stone parallel (left panels) or perpendicular (right panels) to the mainland. In the latter case, the average distance between the islands and the mainland is $d_{\mathrm{c}}=150$ like in the baseline model, which means that the distance between the first (respectively, last) emerging island and the mainland is less (respectively, more) than 150. In both kinds of archipelagos, the distance between neighbour islands is $d_{\mathrm{i}}=20$ like in the baseline model, which means that the distance between non-neighbour islands is more than 20. The other parameters are set to default values (Table S2.1). 

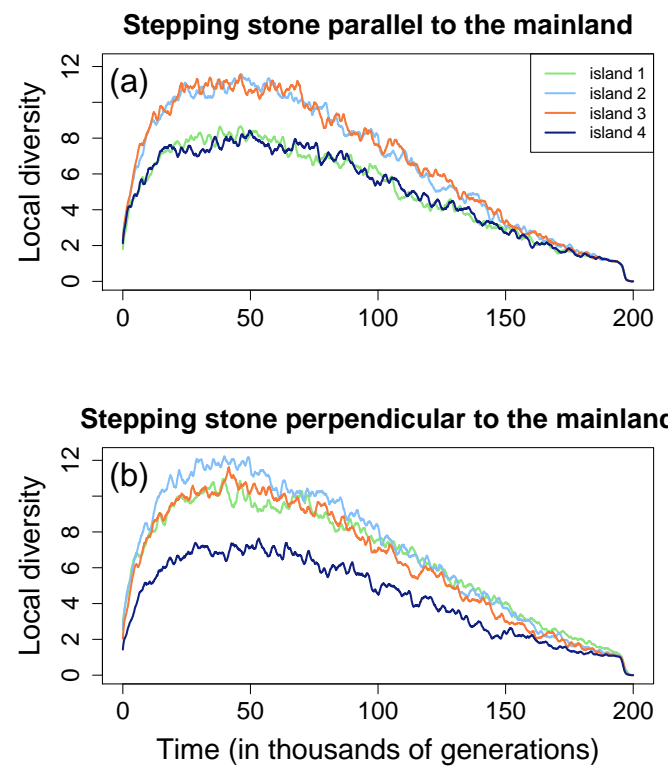

Figure S2.10: Mean dynamics over 25 replicate simulations of local diversity in an archipelago shaped as a linear stepping stone parallel (a) or perpendicular (b) to the mainland. In both cases, all the islands appear simultaneously at time 0 (i.e. $t_{\text {inter }}=0$ ). In the stepping stone perpendicular to the mainland, the average distance between the islands and the mainland is $d_{\mathrm{c}}=150$ like in the baseline model, which means that the distance between the first (respectively, last) emerging island and the mainland is less (respectively, more) than 150 . In both kinds of archipelagos, the distance between neighbour islands is $d_{\mathrm{i}}=$ 20 like in the baseline model, which means that the distance between nonneighbour islands is more than 20. The other parameters are set to default values (Table S2.1). 


\section{Appendix S3 Robustness of the conclusions and parameter space exploration}

Our conclusions are necessarily limited by the assumptions of the model, which we intentionally designed to be as simple as possible. In particular, we only considered the geo-environmental dynamics of volcanic oceanic islands. We cannot completely exclude that our conclusions do not hold for other kinds of islands, but this should be carefully checked. The assumption of ecological neutrality within the archipelago is obviously another limitation. This assumption implies that our conclusions can be reasonably applied to a given clade, with species with comparable ecological characteristics, but not to an entire island community. The neutrality assumption was necessary to properly understand the archipelago effect combined with the history effect. Understanding how these effects interact with ecologically non-neutral dynamics is a next step, which could be done for example by combining our approach with the one of Cabral et al. (2019a) and Cabral et al. (2019b) who explicitly considered ecologically non-neutral processes in a single isolated dynamic island.

Diversity dynamics are variable between replicate simulations (Fig. S3.11). This means that empirical observations in any particular archipelago may differ from the average trends shown in the main text. However, the conclusions drawn from our analysis of the mean dynamics of diversity remain valid when considering variability between replicate simulations. For example, when there is an archipelago effect, the dynamics of the range of local diversity values corresponding to $80 \%$ of the replicate simulations of the baseline model do almost not overlap the range of local diversity values corresponding to $80 \%$ of the replicate simulations of the single isolated island model (Fig. S3.11b). Therefore, our conclusions are robust provided that several archipelagos are analysed together.

We varied all parameters of the model to evaluate their influence on community dynamics. The main text shows the effect of varying the distance between islands $\left(d_{\mathrm{i}}\right.$; Fig. $4 \mathrm{a}$ and Fig. S2.6; the values explored ranged from 5 to 200$)$, of varying the time interval between island emergence $\left(t_{\text {inter }}\right.$; Fig. $4 \mathrm{~b}$ and Fig. S2.7; the values explored ranged from 0 to 1.5.10 ${ }^{5}$ ) and of varying the distance to the mainland $\left(d_{\mathrm{c}}\right.$; Fig. $4 \mathrm{c}$ and Fig. S2.8; the values explored ranged from 50 to 2,000). Below we show the effect of varying the other parameters of the model. For each of these other parameters, we show the dynamics of local diversity, of total diversity and of the proportion of all endemic species for one value higher than the one used in the baseline model and for one value lower. The higher and lower values are chosen such that they show typical results among all the values tested. We mention the range of numerical values tested in the text. When necessary, we show other outputs that are useful to understand the observed biodiversity dynamics. The only exception is the parameter $\beta$, the per-capita birth rate: because the value of this parameter scales time (Appendix S1), all parameters and outputs are measured in relation to the value of $\beta$. Thus it does thus not make sense to look at the effect of variations of $\beta$. 

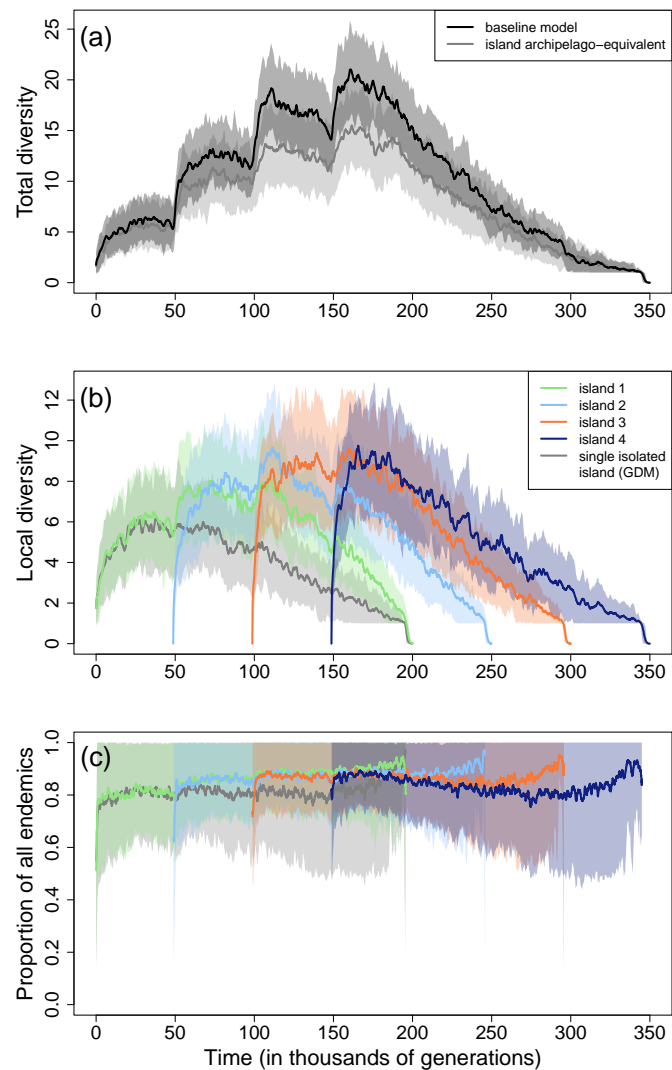

Figure S3.11: Dynamics of diversity at the scale of the archipelago (a), diversity on each island (b), and the proportion of all endemic species (single- and multiple-island endemics) on each island (c) in the baseline model and in the null models. Solid curves show the mean over 25 replicate simulations of the outputs, and shades show the range of values corresponding to $80 \%$ of the replicate simulations. Default parameter values (Table S2.1).

Total number of islands $(\boldsymbol{n})$. The values explored ranged from 1 to 14 . When there are few islands in the archipelago (Fig. S3.12, left columns), the pool of inter-island migrants is small and little diversified. The archipelago effect is thus weak and diversity remains limited. When there are many islands in the archipelago (Fig. S3.12, right columns), the archipelago effect is strong and diversity is higher. With eight islands emerging successively at regular time intervals, some of the intermediate islands are equivalent in the sense that they live in an archipelago with the same structure (as witnessed by the regular variations in the total size of the archipelago, Fig. S3.12h). These islands are subject to the same archipelago effect and thus show similar biodiversity dynamics (Fig. S3.12d).

Rate of emigration $(\boldsymbol{\mu})$. The values explored ranged from 0.5 to 10 (emigration from the mainland) and from $10^{-3}$ to 5 (emigration from an island). We keep the same ratio of the emigration rates from the mainland and from an island as in the baseline model to model the syndrome of loss of dispersal abilities in island species. For a higher emigration rate, the proportion of endemic species is lower (Fig. S3.13f) because of more frequent dispersal from 

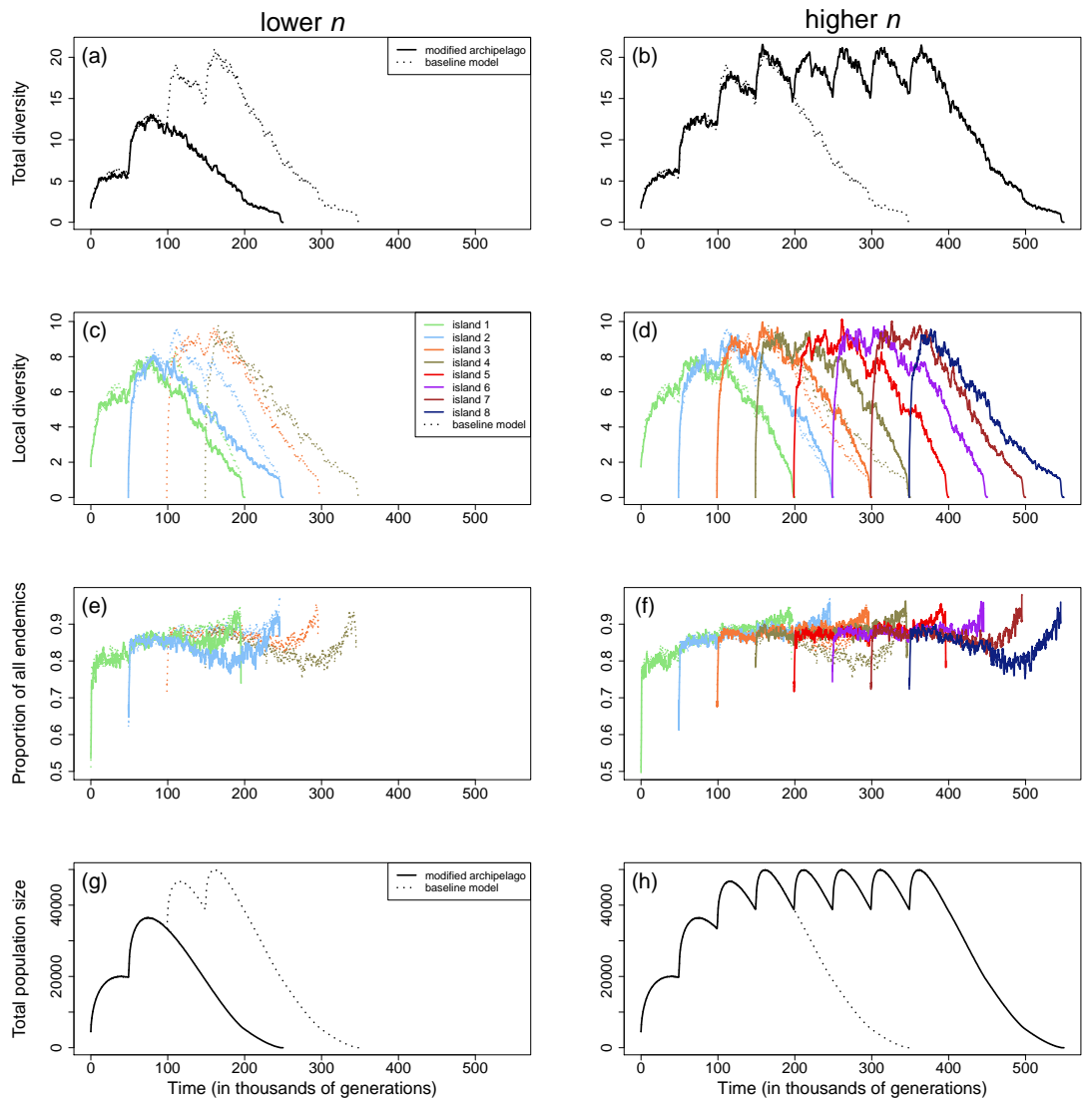

Figure S3.12: Mean dynamics over 25 replicate simulations of various outputs (see Section 2.6 in the main text) in an archipelago with fewer islands (left panels; $n=2$ ) and more islands (right panels; $n=8$ ) than in the baseline model. The other parameters are set to default values (Table S2.1). The outputs are plotted for the archipelago with the modified value of $n$ and with the value of the baseline model.

the mainland, and diversity is higher (Fig. S3.13b,d) due to more frequent speciation events by anagenesis following mainland dispersal and inter-island dispersal (Fig. S3.13h,j,l). The dynamics of biodiversity are more different between islands for a higher value of $\mu$ (Fig. S3.13d), which indicates a larger contribution of the archipelago effect to explain diversity dynamics. This is because the distance between islands in an archipelago is shorter than the distance between the islands and the mainland. Dispersal is thus more likely to be successful between islands than from the mainland, so that an increase in the emigration rate benefits inter-island dispersal (and hence the archipelago effect) more than mainland dispersal.

Individual dispersal ability (c). The values explored ranged from 5 to 100 (individuals emigrating from the mainland) and from 1 to 40 (individuals emigrating from an island). We keep the same ratio of the dispersal ability of individuals from the mainland and from an island as in the baseline model to model the syndrome of loss of dispersal abilities in island species. Given that in the baseline model, the dispersal ability of individuals from islands is close to the distance between islands $\left(c=10\right.$ and $\left.d_{\mathrm{i}}=20\right)$, increasing the 

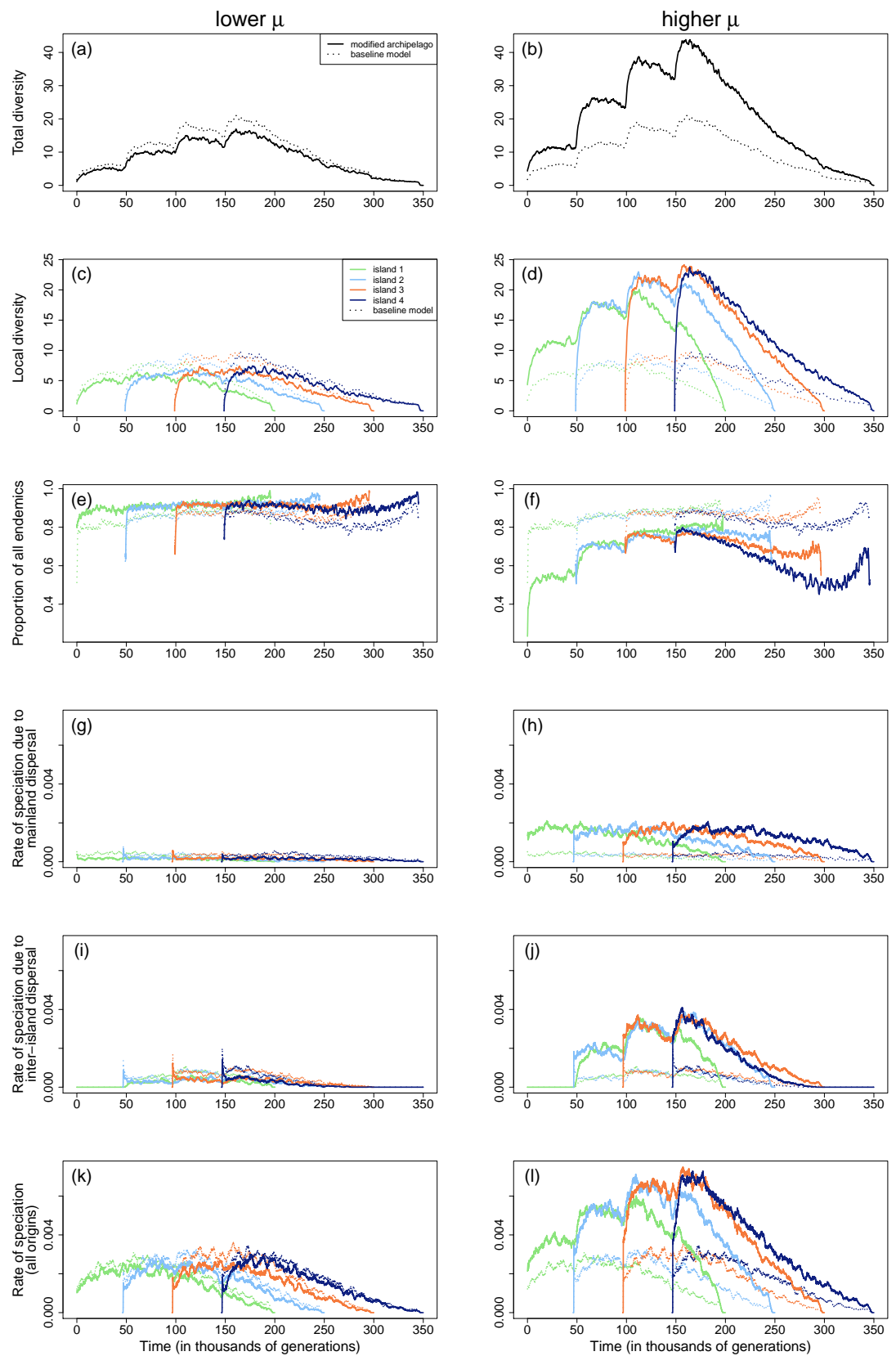

Figure S3.13: Mean dynamics over 25 replicate simulations of various outputs (see Section 2.6 in the main text) in an archipelago with a lower value of $\mu$ (left panels; $\mu=0.5$ for emigration from the mainland and $\mu=0.05$ for emigration from an island) and a higher value (right panels; $\mu=5$ for emigration from the mainland and $\mu=0.5$ for emigration from an island) than in the baseline model. The other parameters are set to default values (Table S2.1). The outputs are plotted for the archipelago with the modified value of $\mu$ and with the value of the baseline model.

dispersal ability does not significantly alter inter-island dispersal (Fig. S3.14j). However, it significantly increases dispersal from the mainland (Fig. S3.14h), resulting in a smaller proportion of endemic species (Fig. S3.14f) and higher diversity (Fig. S3.14b,d). When dispersal abilities are reduced compared to the baseline model, dispersal from the mainland is reduced (Fig. S3.14g), leading 
to a bigger proportion of endemic species (Fig. S3.14e). Inter-island dispersal is also reduced (Fig. S3.14i), leading to a lower speciation rate (Fig. S3.14k). Overall, these effects reduce diversity (Fig. S3.14a,c).
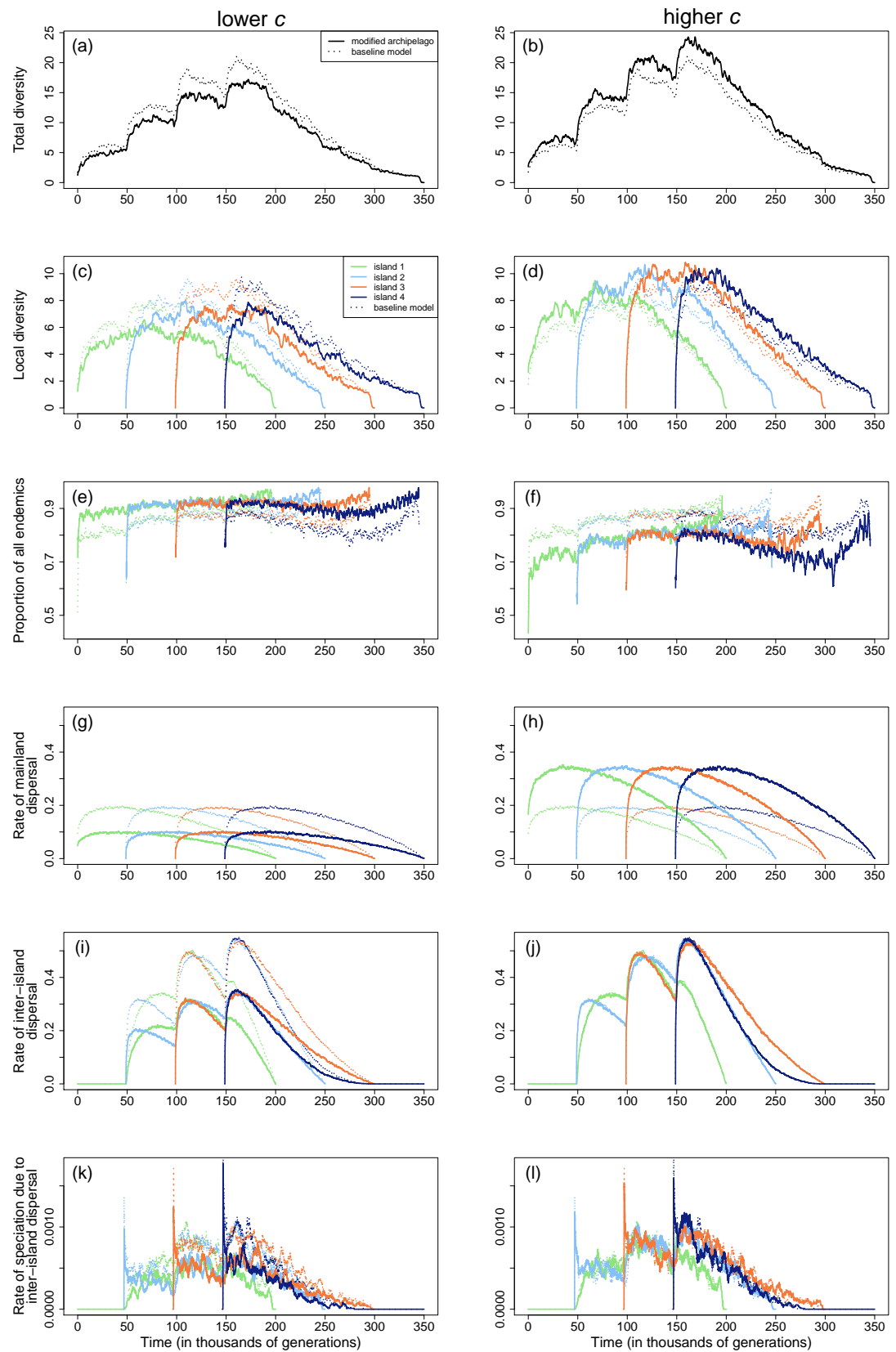

Figure S3.14: Mean dynamics over 25 replicate simulations of various outputs (see Section 2.6 in the main text) in an archipelago with a lower value of $c$ (left panels; $c=12.5$ for individuals emigrating from the mainland and $c=5$ for individuals emigrating from an island) and a higher value (right panels; $c=50$ for individuals emigrating from the mainland and $c=20$ for individuals emigrating from an island) than in the baseline model. The other parameters are set to default values (Table S2.1). The outputs are plotted for the archipelago with the modified value of $c$ and with the value of the baseline model. 
Strength of density-dependence of mortality $(\gamma)$. The values explored ranged from $10^{-5}$ to 5 . A lower value of $\gamma$ is expected to more easily allow deviation from demographic equilibrium. For a low value of $\gamma$ the populations indeed do not reach equilibrium (Fig. S3.15g). Because population size almost always remains below the demographic equilibrium, species richness is lower than in the baseline model (Fig. S3.15a,c).
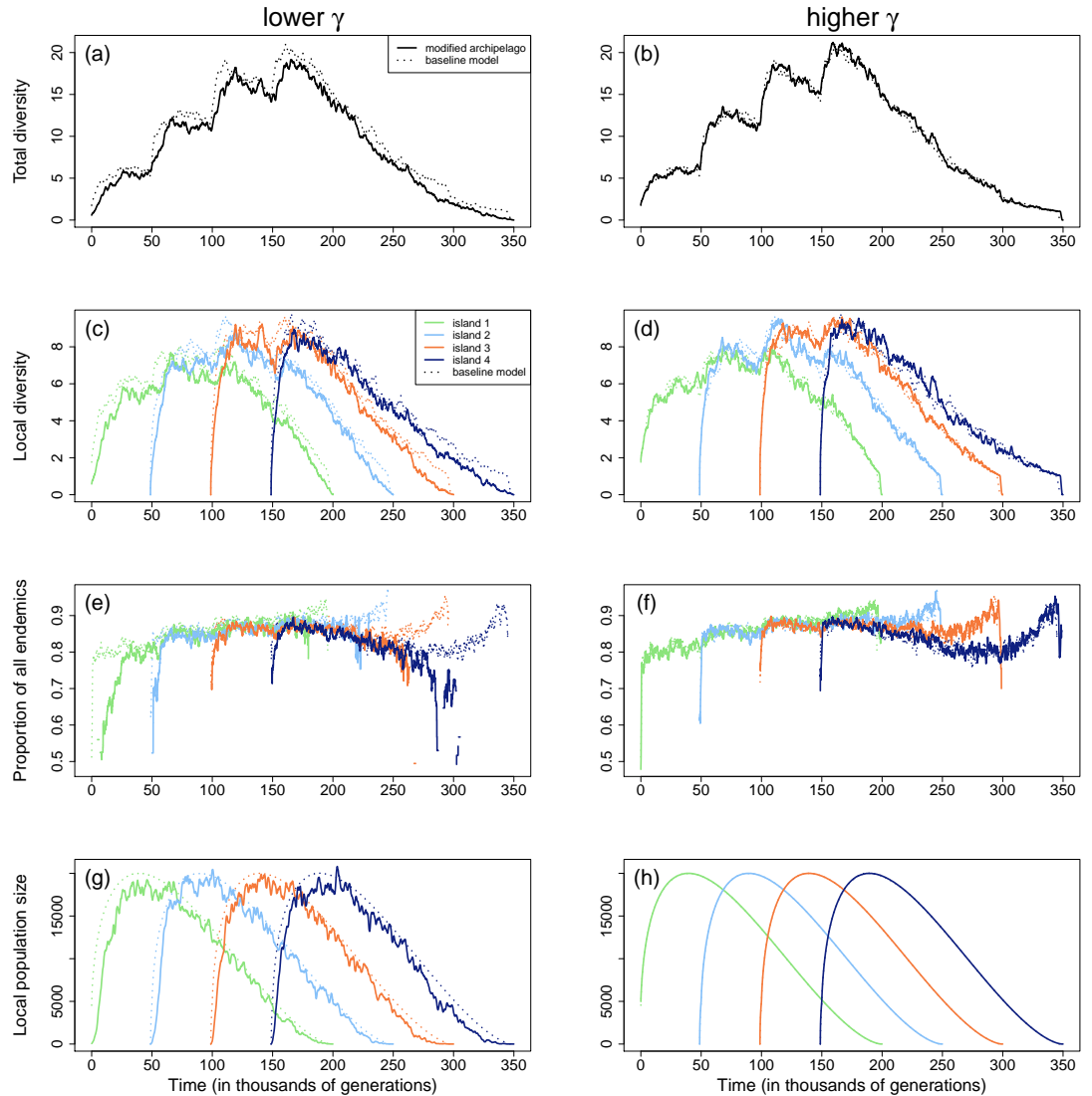

Figure S3.15: Mean dynamics over 25 replicate simulations of various outputs (see Section 2.6 in the main text) in an archipelago with a lower value of $\gamma$ (left panels; $\gamma=10^{-3}$ ) and a higher value (right panels; $\gamma=1$ ) than in the baseline model. The other parameters are set to default values (Table S2.1). The outputs are plotted for the archipelago with the modified value of $\gamma$ and with the value of the baseline model.

Rate of cladogenesis initiation $(\sigma)$. The values explored ranged from $10^{-7}$ to $10^{-3}$. With a higher value of $\sigma$, cladogenesis is initiated more often. The corresponding rate of speciation is higher (Fig. S3.16h), which results in a greater diversity and a higher proportion of endemic species (Fig. S3.16b,d,f). Because cladogenesis has the same dynamics on all islands (it depends on population size, and all islands have the same carrying capacity function) and because for higher values of $\sigma$ cladogenesis becomes the main process at the origin of diversity, diversity dynamics tend to look similar on all islands (Fig. S3.16d), i.e. the relative contribution of the archipelago effect in explaining diversity dynamics diminishes. 

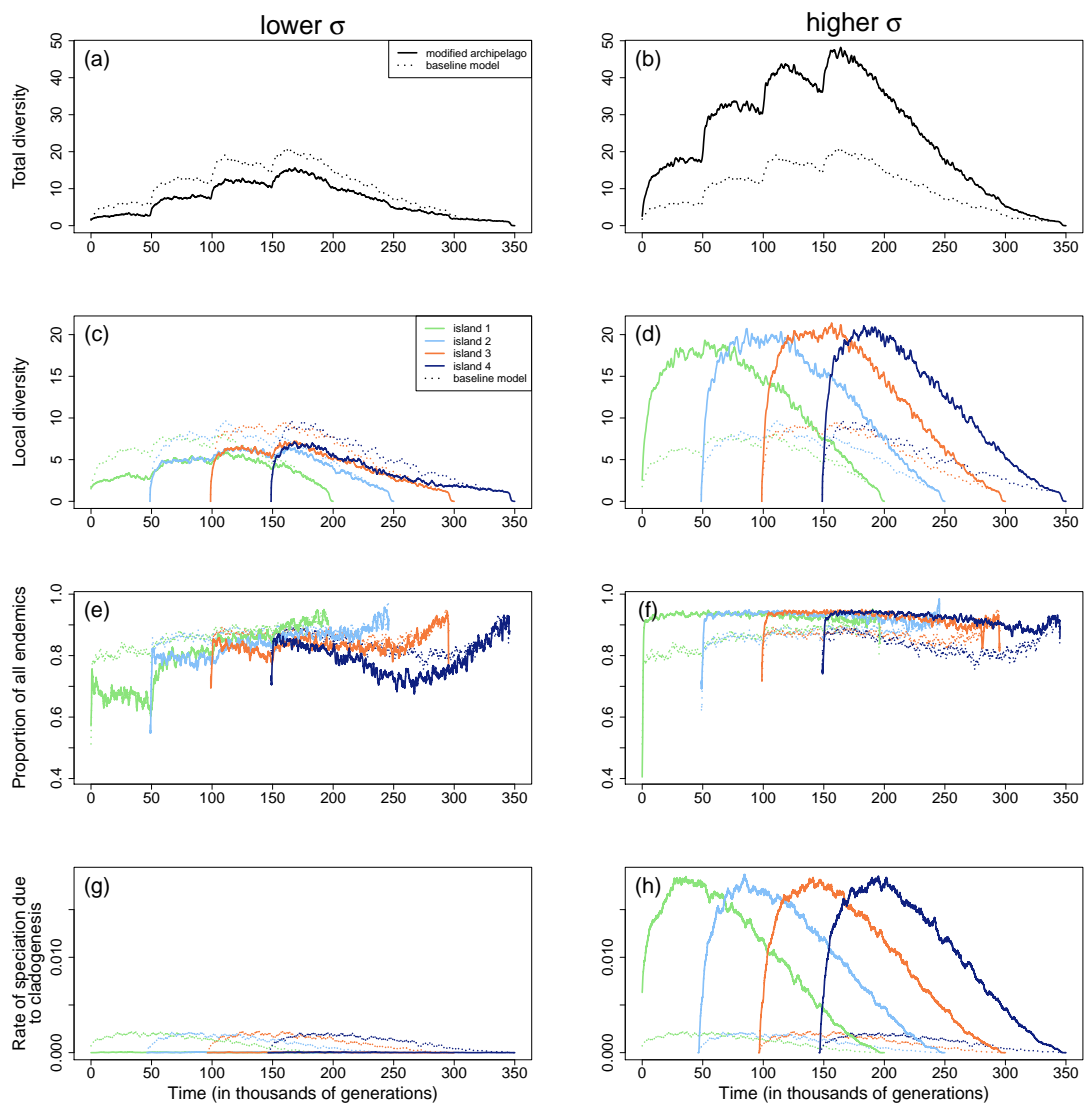

Figure S3.16: Mean dynamics over 25 replicate simulations of various outputs (see Section 2.6 in the main text) in an archipelago with a lower value of $\sigma$ (left panels; $\sigma=10^{-6}$ ) and a higher value (right panels; $\sigma=5.10^{-4}$ ) than in the baseline model. The other parameters are set to default values (Table S2.1). The outputs are plotted for the archipelago with the modified value of $\sigma$ and with the value of the baseline model.

Minimal duration for speciation $(\boldsymbol{\tau})$. The values explored ranged from 100 to 5,000 . When the minimum duration for speciation is longer, speciation fails more easily (Fig. S3.17l) because of extinction of the diverging populations before speciation is achieved. Consequently, the archipelago effect is weaker (Fig. S3.17j) despite the unchanged quantity of inter-island migrants (Fig. S3.17h). This reduces both local and total diversity and the proportion of endemic species (Fig. S3.17b,d,f). In brief, the archipelago effect is weaker due to the reduced diversity of the pool of inter-island migrants.

Strength with which dispersal delays speciation $(\boldsymbol{\alpha})$. The values explored ranged from 1 to 5000. Compared to the baseline model, a larger value of $\alpha$ delays more speciation (Fig. S3.18j), which thus succeeds at a lower rate (Fig. S3.18h). The archipelago effect is consequently reduced. However, in the range of values explored here, the consequences for biodiversity dynamics are limited (Fig. S3.18a-f).

Mainland metacommunity size $(\boldsymbol{J})$. The values explored ranged from $10^{4}$ to $10^{8}$. When the size of the mainland increases, mainland dispersal in- 

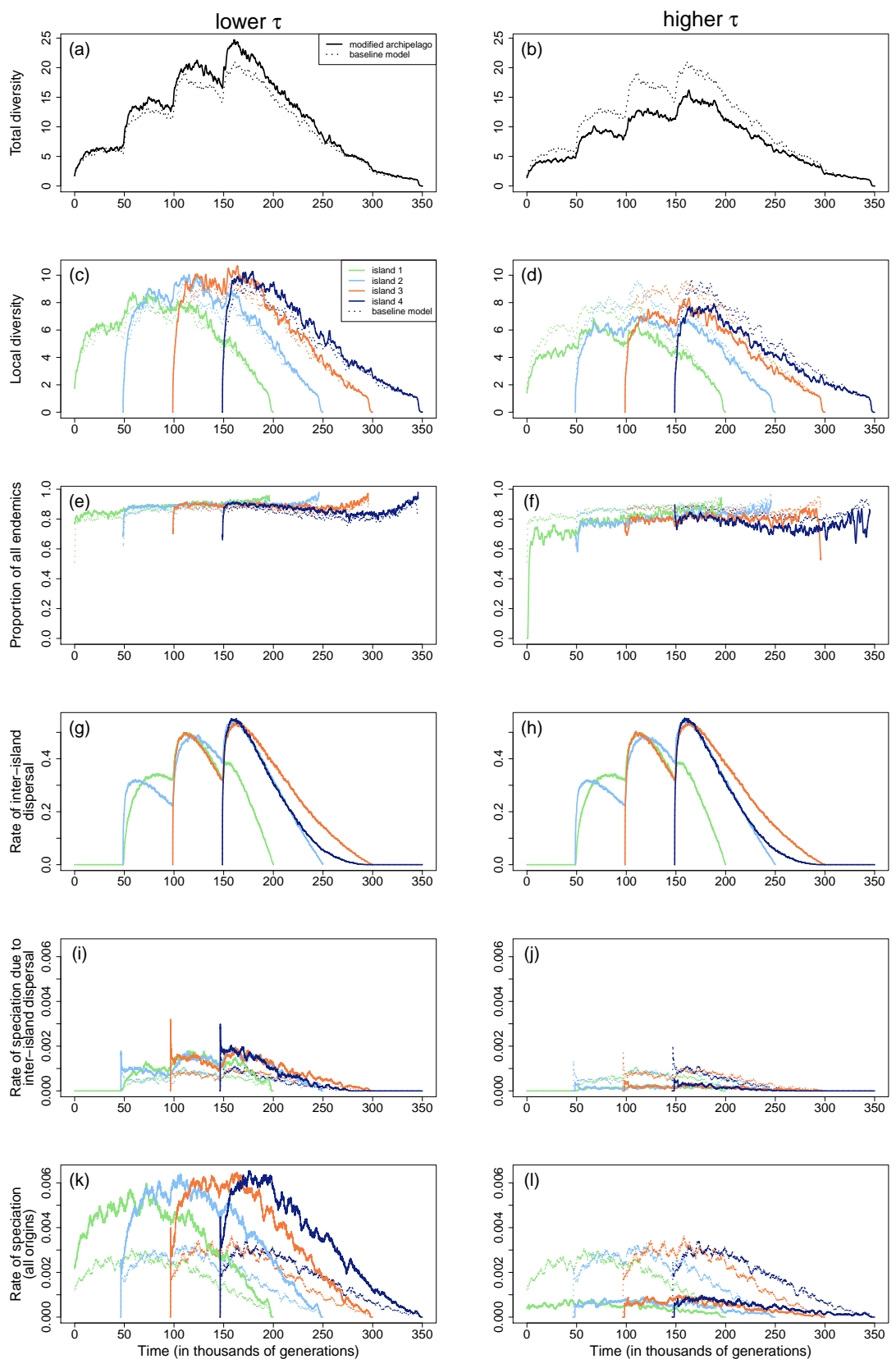

Figure S3.17: Mean dynamics over 25 replicate simulations of various outputs (see Section 2.6 in the main text) in an archipelago with a lower value of $\tau$ (left panels; $\tau=250$ ) and a higher value (right panels; $\tau=2000$ ) than in the baseline model. The other parameters are set to default values (Table S2.1). The outputs are plotted for the archipelago with the modified value of $\tau$ and with the value of the baseline model.

creases, which reduces the proportion of endemic species (Fig. S3.19f). Combined with increased diversity of mainland migrants, this leads to a higher rate of speciation due to mainland dispersal (Fig. S3.19h). By contrast, the archipelago effect does not depend on $J$, as evidenced for example by the unchanged speciation rate due to inter-island dispersal (Fig. S3.19i,j). As a result of both processes, total and local diversity increase with increasing $J$ (Fig. S3.19a-d) but the relative contribution of the archipelago effect in ex- 

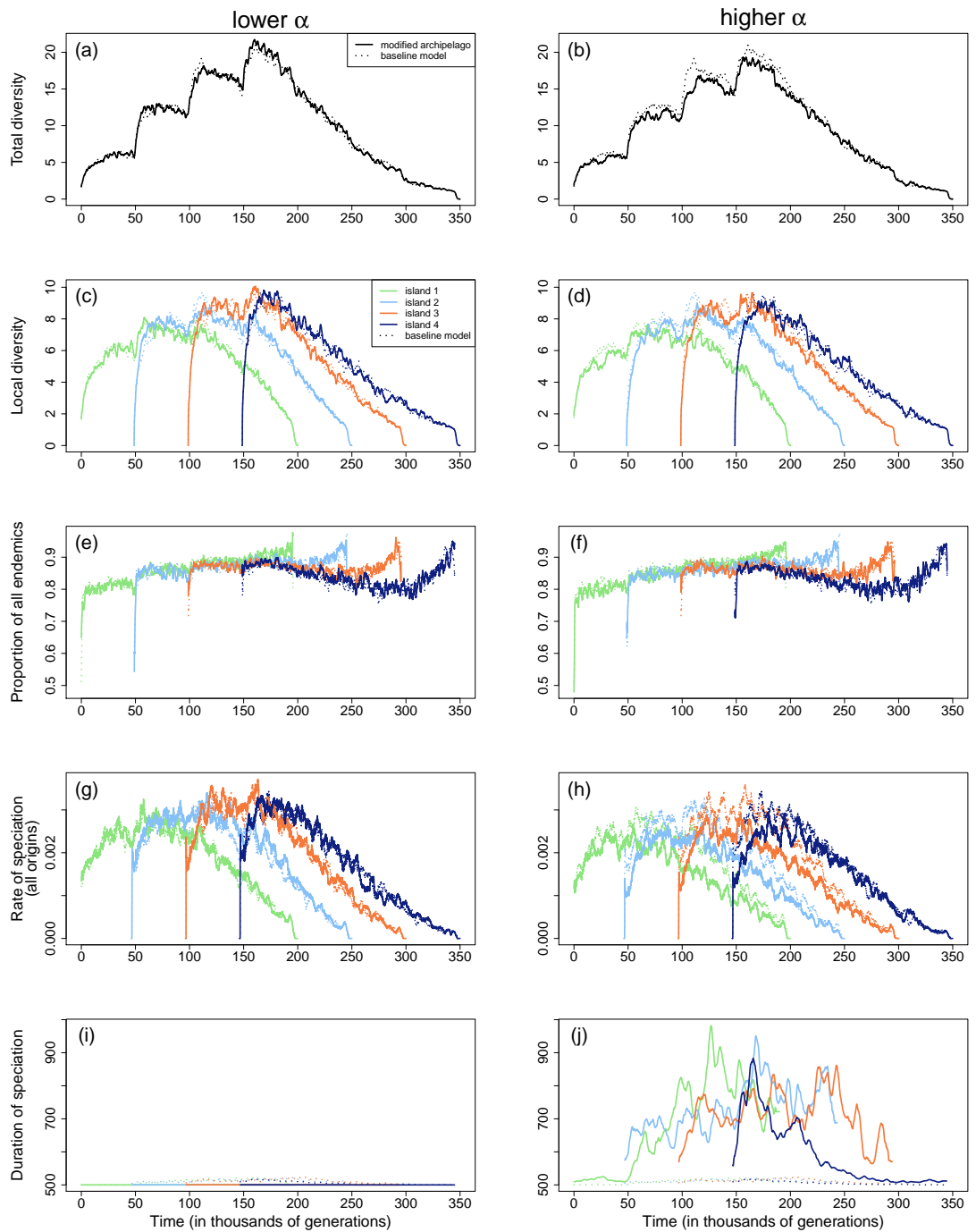

Figure S3.18: Mean dynamics over 25 replicate simulations of various outputs (see Section 2.6 in the main text) in an archipelago with a lower value of $\alpha$ (left panels; $\alpha=1$ ) and a higher value (right panels; $\alpha=5000$ ) than in the baseline model. The other parameters are set to default values (Table S2.1). The outputs are plotted for the archipelago with the modified value of $\alpha$ and with the value of the baseline model.

plaining diversity dynamics is lower at higher values of $J$, which explains why the local biodiversity dynamics are more similar between islands (Fig. S3.19d) compared to the dynamics with a lower value of $J$ (Fig. S3.19c) .

Mainland fundamental biodiversity number $(\boldsymbol{\theta})$. The values explored ranged from 10 to 100 . With a higher value of $\theta$, the mainland is more diverse so that migrants from the mainland are more likely to be new to the archipelago (Fig. S3.20g,h), which tends to increase diversity in the archipelago. However, this effect is offset by a higher species extinction rate (Fig. S3.20i,j), leading to diversity dynamics that remain unchanged compared to the baseline model (Fig. S3.20a-f). The value of $\theta$ does not affect the archipelago effect. 

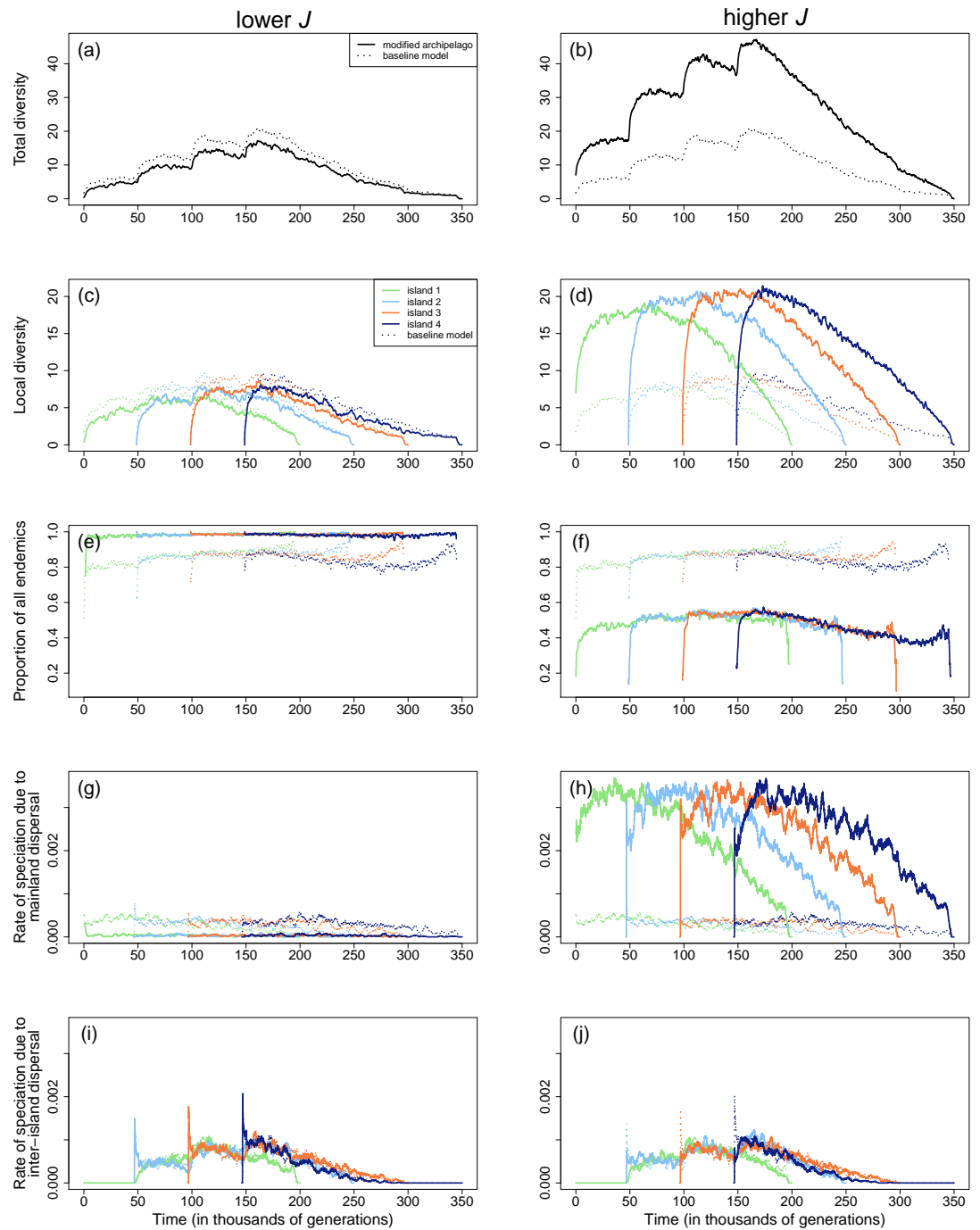

Figure S3.19: Mean dynamics over 25 replicate simulations of various outputs (see Section 2.6 in the main text) in an archipelago with a lower value of $J$ (left panels; $J=10^{5}$ ) and a higher value (right panels; $J=10^{7}$ ) than in the baseline model. The other parameters are set to default values (Table S2.1). The outputs are plotted for the archipelago with the modified value of $J$ and with the value of the baseline model.

Lifetime of an island $\left(\boldsymbol{t}_{\text {life }}\right)$. The values explored ranged from $2.10^{4}$ to $4.10^{5}$. To separate the effect of the lifetime of an island from all other factors, here we covary $t_{\text {life }}$ and $t_{\text {inter }}$ such that islands coexist for the same proportion of their life as in the baseline model. The results in Fig. S3.21 show that, in this case, varying $t_{\text {life }}$ only has a scaling effect on the time axis.

Maximum island carrying capacity $\left(\boldsymbol{K}_{\max }\right)$. The values explored ranged from $5.10^{3}$ to $4.10^{4}$. As expected, the value of $K_{\max }$ scales diversity and the proportion of endemic species (Fig. S3.22).

Proportion of the island lifetime at which the maximum carrying capacity is reached $\left(\boldsymbol{p}_{\max }\right)$. The values explored ranged from 0.1 to 0.3. 

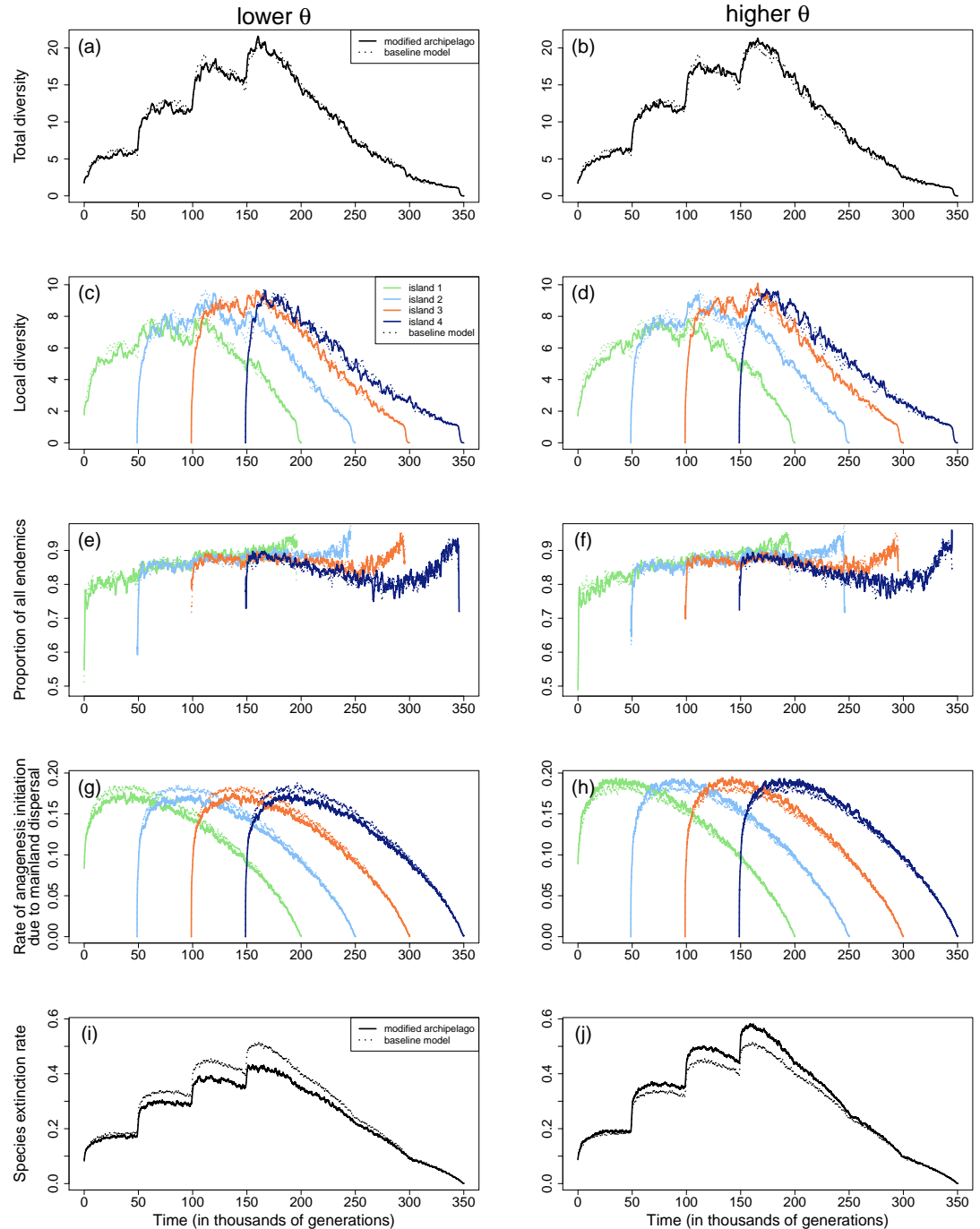

Figure S3.20: Mean dynamics over 25 replicate simulations of various outputs (see Section 2.6 in the main text) in an archipelago with a lower value of $\theta$ (left panels; $\theta=10$ ) and a higher value (right panels; $\theta=100$ ) than in the baseline model. The other parameters are set to default values (Table S2.1). The outputs are plotted for the archipelago with the modified value of $\theta$ and with the value of the baseline model.

A shift in the time at which the islands reach their maximum carrying capacity results in a proportional shift in the time at which diversity reaches maximum (Fig. S3.23). 

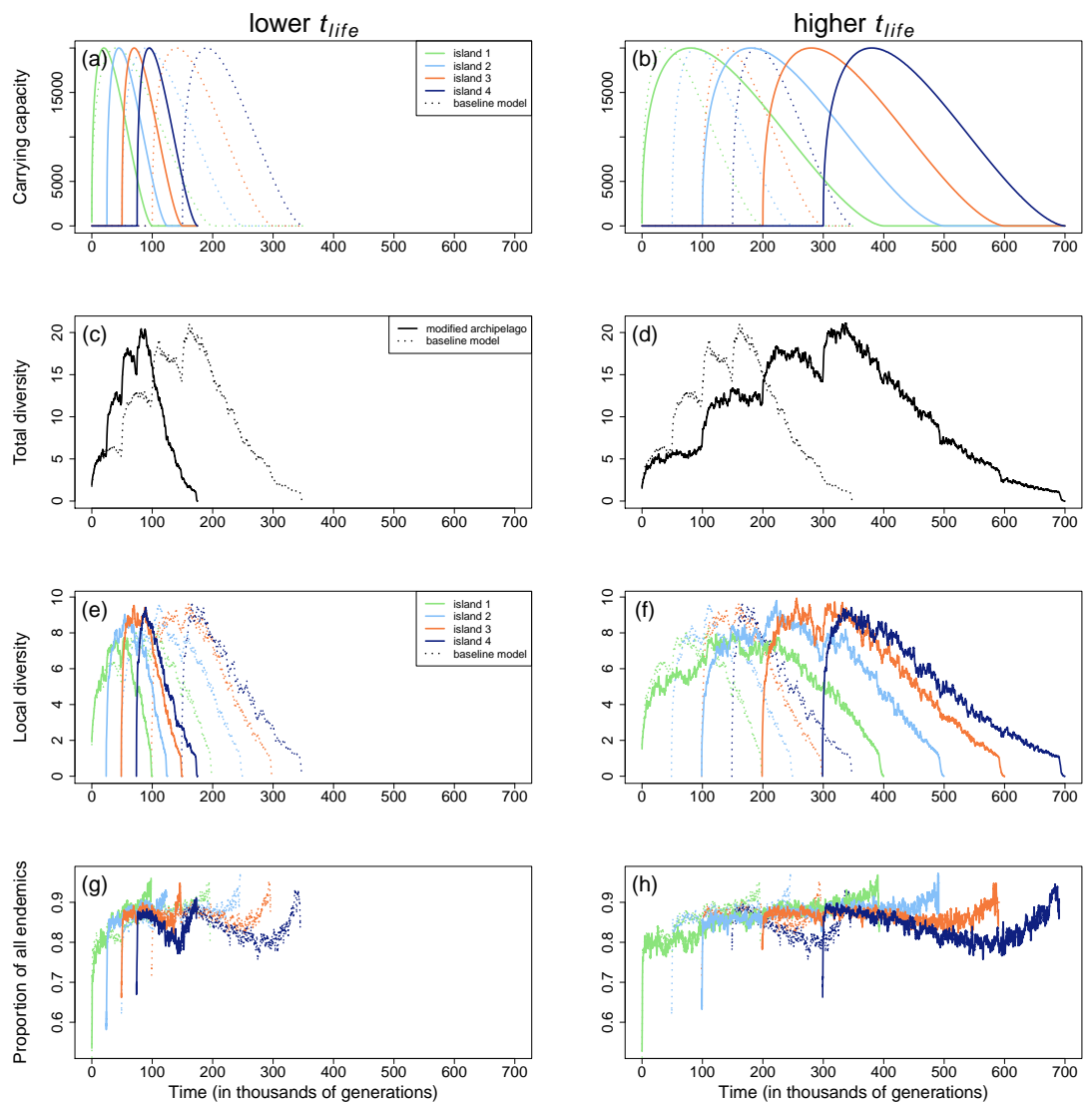

Figure S3.21: Mean dynamics over 25 replicate simulations of various outputs (see Section 2.6 in the main text) in an archipelago with a lower value for $t_{\text {life }}$ and $t_{\text {inter }}$ (left panels; $t_{\text {life }}=10^{5}$ and $t_{\text {inter }}=2.5 .10^{4}$ ) and a higher value (right panels; $t_{\text {life }}=4.10^{5}$ and $t_{\text {inter }}=10^{5}$ ) than in the baseline model. The other parameters are set to default values (Table S2.1). The outputs are plotted for the archipelago with the modified values of $t_{\text {life }}$ and $t_{\text {inter }}$, and with the ones of the baseline model. 

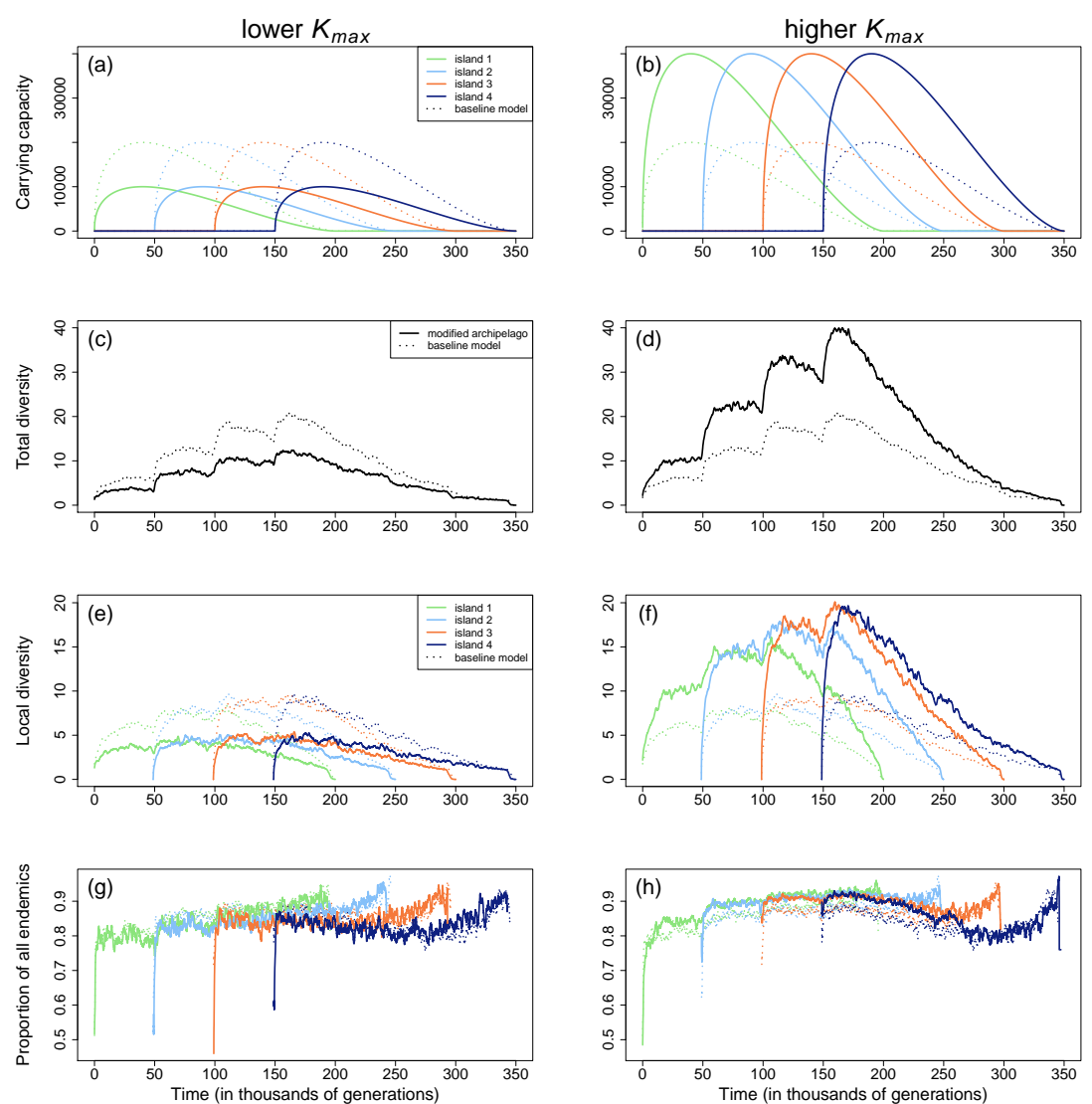

Figure S3.22: Mean dynamics over 25 replicate simulations of various outputs (see Section 2.6 in the main text) in an archipelago with a lower value of $K_{\max }$ (left panels; $K_{\max }=10^{4}$ ) and a higher value (right panels; $K_{\max }=4.10^{4}$ ) than in the baseline model. The other parameters are set to default values (Table S2.1). The outputs are plotted for the archipelago with the modified value of $K_{\max }$ and with the value of the baseline model. 

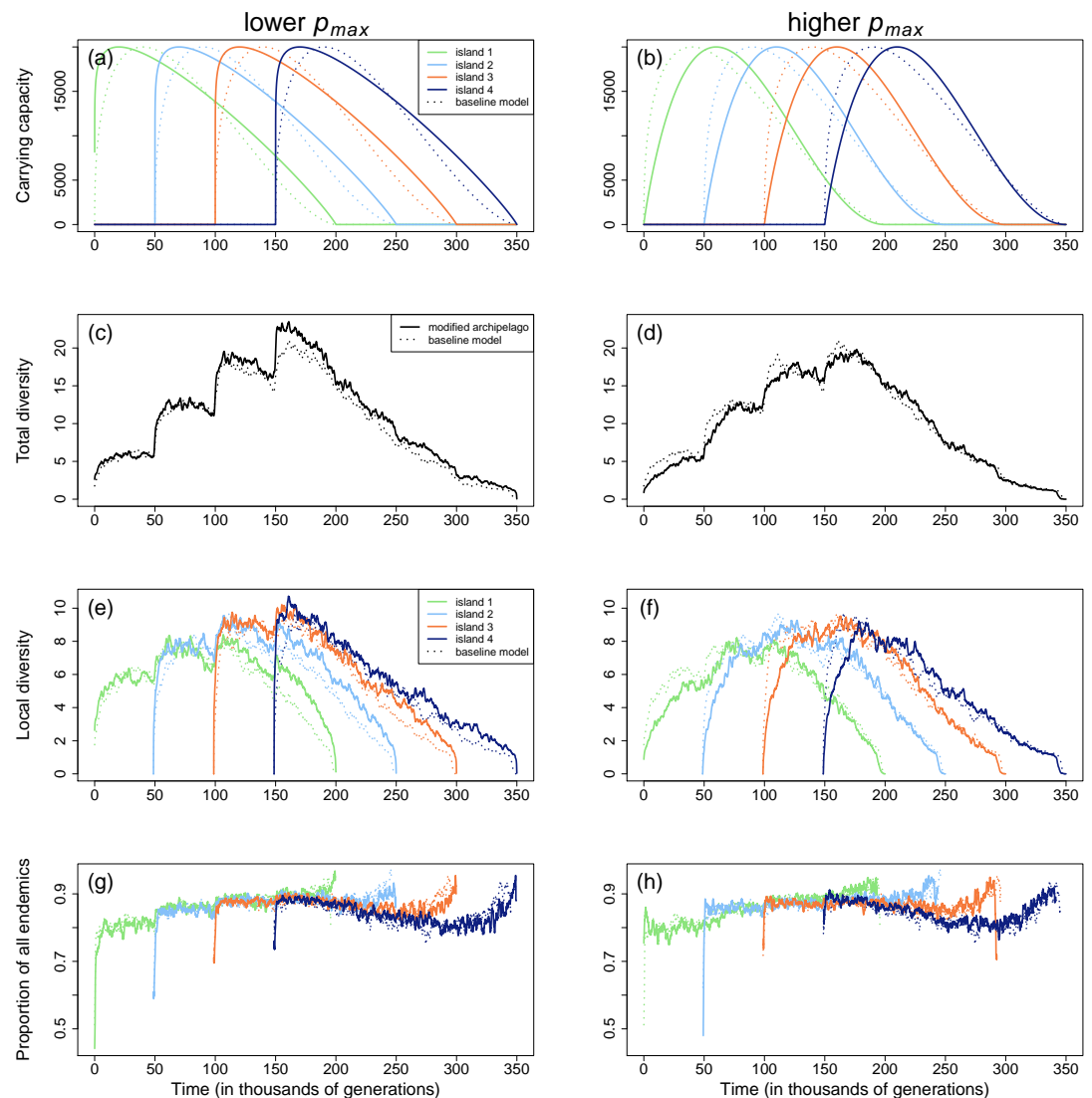

Figure S3.23: Mean dynamics over 25 replicate simulations of various outputs (see Section 2.6 in the main text) in an archipelago with a lower value of $p_{\max }$ (left panels; $p_{\max }=0.1$ ) and a higher value (right panels; $p_{\max }=0.3$ ) than in the baseline model. The other parameters are set to default values (Table S2.1). The outputs are plotted for the archipelago with the modified value of $p_{\max }$ and with the value of the baseline model. 


\section{References}

Cabral, J. S., Whittaker, R. J., Wiegand, K., and Kreft, H. (2019a). Assessing predicted isolation effects from the general dynamic model of island biogeography with an eco-evolutionary model for plants. J Biogeogr, 46:1569-1581.

Cabral, J. S., Wiegand, K., and Kreft, H. (2019b). Interactions between ecological, evolutionary, and environmental processes unveil complex dynamics of island biodiversity. J Biogeogr, 46:1582-1597.

Clark, J. S., Silman, M., Kern, R., Macklin, E., and HilleRisLambers, J. (1999). Seed dispersal near and far: Patterns across temperate and tropical forest. Ecology, 80:1475-1494.

Galassi, M., Davies, J., Theiler, J., Gough, B., Jungman, G., Alken, P., Booth, M., and Rossi, F. (2009). GNU Scientific Library Reference Manual - Third Edition.

Gascuel, F., Laroche, F., Bonnet-Lebrun, A.-S., and Rodrigues, A. S. (2016). The effects of archipelago spatial structure on island diversity and endemism: predictions from a spatially-structured neutral model. Evolution, 70(11):2657-2666.

Gillespie, D. T. (1977). Exact stochastic simulation of coupled chemical reactions. J Phys Chem, 81:2340-2361.

Gilpin, M. E. and Diamond, J. M. (1976). Calculation of immigration and extinction curves from the species-area-distance relation. Proc Natl Acad Sci USA, 73(11):4130-4134.

Hankin, R. (2009). R Package 'untb': Ecological Drift Under the UNTB.

MacArthur, R. H. and Wilson, E. O. (1967). The Theory of Island Biogeography. Princeton University Press, Princeton, NJ.

R Core Team (2017). R: A Language and Environment for Statistical Computing. R Foundation for Statistical Computing, Vienna, Austria.

Valente, L., Etienne, R., and Phillimore, A. (2014). The effects of island ontogeny on species diversity and phylogeny. $P$ Roy Soc Lond B Bio, 281:20133227. 